\title{
THE FIRST GALAXIES: ASSEMBLY WITH BLACK HOLE FEEDBACK
}

\author{
Myoungwon Jeon ${ }^{1}$, Andreas H. Pawlik ${ }^{1}$, Thomas H. Greif ${ }^{2}$, Simon C. O. Glover ${ }^{3}$, Volker Bromm ${ }^{1,2}$, \\ Miloš Milosavljević ${ }^{1}$, and Ralf S. Klessen ${ }^{3}$ \\ ${ }^{1}$ Department of Astronomy and Texas Cosmology Center, University of Texas, Austin, TX 78712, USA; myjeon@astro.as.utexas.edu \\ ${ }^{2}$ Max-Planck-Institut für Astrophysik, Karl-Schwarzschild-Strasse 1, 85740 Garching bei München, Germany \\ ${ }^{3}$ Zentrum für Astronomie der Universität Heidelberg, Institut für Theoretische Astrophysik, Albert-Ueberle-Strasse 2, 69120 Heidelberg, Germany \\ Received 2011 November 23; accepted 2012 April 25; published 2012 July 3
}

\begin{abstract}
We study how the first galaxies were assembled under feedback from the accretion onto a central black hole (BH) that is left behind by the first generation of metal-free stars through self-consistent, cosmological simulations. X-ray radiation from the accretion of gas onto BH remnants of Population III (Pop III) stars, or from high-mass X-ray binaries (HMXBs), again involving Pop III stars, influences the mode of second generation star formation. We track the evolution of the $\mathrm{BH}$ accretion rate and the associated X-ray feedback starting with the death of the Pop III progenitor star inside a minihalo and following the subsequent evolution of the $\mathrm{BH}$ as the minihalo grows to become an atomically cooling galaxy. We find that X-ray photoionization heating from a stellar-mass $\mathrm{BH}$ is able to quench further star formation in the host halo at all times before the halo enters the atomic cooling phase. X-ray radiation from an HMXB, assuming a luminosity close to the Eddington value, exerts an even stronger, and more diverse, feedback on star formation. It photoheats the gas inside the host halo, but also promotes the formation of molecular hydrogen and cooling of gas in the intergalactic medium and in nearby minihalos, leading to a net increase in the number of stars formed at early times. Our simulations further show that the radiative feedback from the first BHs may strongly suppress early BH growth, thus constraining models for the formation of supermassive BHs.
\end{abstract}

Key words: black hole physics - cosmology: observations - galaxies: formation - galaxies: high-redshift - $\mathrm{H}$ II regions - hydrodynamics - intergalactic medium

Online-only material: color figures

\section{INTRODUCTION}

Radiative feedback from the first generation of stars, the so-called Population III (Pop III), is a crucial ingredient in determining how the first protogalaxies assembled, and in setting the initial conditions for subsequent, second-generation star formation (Barkana \& Loeb 2001; Bromm \& Larson 2004; Ciardi \& Ferrara 2005; Bromm et al. 2009). It has been a longstanding goal to understand the formation of the first galaxies subject to different accompanying feedback effects from the first stars, such as ionizing feedback from individual Pop III stars (Ricotti et al. 2002; Abel et al. 2007; Yoshida et al. 2007a), chemical feedback produced by a supernova (SN) explosion (Wise \& Abel 2008; Greif et al. 2010; Wise et al. 2012), and radiative feedback from accreting black holes (BHs; Kuhlen \& Madau 2005; Alvarez et al. 2009; Park \& Ricotti 2011). The radiative feedback from accreting BHs on the formation of the first stars and the assembly of the first galaxies is the focus of the current work.

The first stars are expected to form at a redshift $z \gtrsim 15$ inside dark matter $(\mathrm{DM})$ minihalos with masses of $\sim 10^{6} \widetilde{M_{\odot}}$ (Haiman et al. 1996; Tegmark et al. 1997; Yoshida et al. 2003). Since hydrogen molecules were the only effective low-temperature coolant in the absence of metals, the primordial gas remains relatively warm, with typical temperatures around $200 \mathrm{~K}$. As a consequence of this increased thermal pressure, the first stars are expected to be very massive (Bromm et al. 1999, 2002; Nakamura \& Umemura 2001; Abel et al. 2002; Omukai \& Palla 2003; Yoshida et al. 2006; O'Shea \& Norman 2007). These Pop III stars produce copious amounts of ionizing UV photons, which can photoheat and evacuate the gas residing within the host minihalos (Whalen et al. 2004; Alvarez et al. 2006; Johnson et al. 2007).
After their short lifetime of $\sim 3$ Myr, Pop III stars end their lives in an SN explosion, or the direct collapse into a $\mathrm{BH}$, depending on the progenitor mass (Heger et al. 2003). If the progenitor's mass lies in the range of $15-40 M_{\odot}$, then a conventional core-collapse SN occurs, or, in the case of rapid rotation, a hypernova. The resulting chemical abundance patterns are in good agreement with those observed in extremely metal-poor stars in the Galactic halo (Beers \& Christlieb 2005; Joggerst et al. 2010).

An extreme fate would be a pair-instability supernova (PISN), predicted for progenitor masses between 140 and $260 M_{\odot}$ (Barkat et al. 1967; Heger \& Woosley 2002). A consequence of the very large PISN yields would be that even a single PISN could enrich $\sim 10^{7} M_{\odot}$ of cold, dense gas up to $10^{-4}-10^{-3} Z_{\odot}$ (Karlsson et al. 2008; Greif et al. 2010). Such high metallicities would be in excess of any predicted critical value, beyond which a transition of star formation mode from massive Pop III stars to normal Population II (Pop II) would occur (Omukai 2000; Bromm et al. 2001a; Schneider et al. 2002).

For progenitor masses within the range of $40 M_{\odot} \lesssim M \lesssim$ $140 M_{\odot}$ or $M \gtrsim 260 M_{\odot}$, the Pop III star will become a massive $\mathrm{BH}$ via direct gravitational collapse. The ensuing merging of minihalos, provided they contain cold gas, and the smooth accretion of gas from the intergalactic medium (IGM) will feed the central BH. A fraction of the accreted mass energy will then be released as radiative energy, resulting in a miniquasar (Madau \& Rees 2001; Ricotti \& Ostriker 2004; Madau et al. 2004; Kuhlen \& Madau 2005; Wheeler \& Johnson 2011), which will ionize the surrounding neutral medium and heat the pre-galactic gas.

Recent simulations have shown that, even in the absence of metal-line and dust cooling, the primordial gas is able to fragment, leading to a broad initial mass function (IMF) 
including also primordial stars of relatively low mass (Turk et al. 2009; Stacy et al. 2010, 2012; Clark et al. 2011a, 2011b; Greif et al. 2011; Smith et al. 2011; Prieto et al. 2011), which may be organized in binaries or stellar systems of still higher multiplicity. Such a scenario raises the possibility of the presence of high-mass X-ray binaries (HMXB) at $z \gtrsim 10$, i.e., binary systems composed of a BH accreting gas from the surface of its stellar companion (Mirabel et al. 2011; Haiman 2011). HMXBs may provide a much stronger feedback on the formation of the first galaxies than isolated BHs, which have X-ray luminosities that depend crucially on the densities and temperatures of the surrounding gas (Kuhlen \& Madau 2005; Alvarez et al. 2009; Park \& Ricotti 2011).

We should note that the assumed presence of HMXBs at high redshifts sensitively depends on the Pop III IMF and multiplicity. Recent simulations have provided intriguing hints for the Pop III IMF, such that the Pop III stars were likely to form in binaries or multiples rather than in isolation, but any predictions are subject to uncertainties due to the limitations of the sink particle method. A sink particle treatment, which has been commonly adopted in smoothed particle hydrodynamics (SPH) simulations to follow the subsequent evolution of a protostellar core, allows us to avoid the problem of prohibitively small numerical time steps, the so-called Courant myopia (Bate et al. 1995; Bromm et al. 2002; Jappsen et al. 2005). The resulting protostellar IMF is affected by the detailed treatment for sink particle mergers and by the ability of such schemes to self-consistently model the interaction between sink particles and the surrounding gas (Greif et al. 2011). It is, therefore, still an open question how the early protostellar mass function will be mapped into the final IMF of Pop III stars.

In this paper, we investigate the feedback effects from such accreting stellar-mass BHs by focusing on the question: "How does a stellar black hole, a remnant of a Pop III star, influence the subsequent star formation and in turn the assembly process of the first galaxies?" Our work is complementary to Greif et al. (2010) because our cosmological simulations start from the same initial conditions. The difference is that we include the feedback from accreting BHs while in their work the feedback from a PISN was taken into account. We can thus investigate how the assembly process of the first galaxies differs in response to different assumptions regarding the fate of the first stars and the accompanying feedback. In addition, we can constrain the resulting gas properties inside the centers of such primordial galaxies, thus providing us with the initial conditions for secondgeneration star formation.

It is challenging to unambiguously define the first galaxies (e.g., Bromm \& Yoshida 2011). A plausible approach is to posit that the first galaxies should be massive enough to exhibit a sufficiently deep potential well to retain gas that is photoheated by stars or BHs. Another criterion is that a galaxy should sustain a self-regulated star formation mode. Recent cosmological simulations suggest that the first galaxies resided in the so-called atomic cooling halos (Oh \& Haiman 2002) with typical masses of $\sim 10^{8} M_{\odot}$ at $z \sim 10$ (Wise \& Abel 2007, 2008; Greif et al. $2008,2010)$. Whether the presence of an X-ray radiation field such as those produced by accreting BHs will result in positive or negative feedback on the formation of these galaxies remains an open key question (Oh 2001; Ricotti et al. 2001; Glover \& Brand 2003; Machacek et al. 2003; Ricotti \& Ostriker 2004; Kuhlen \& Madau 2005; Zaroubi et al. 2007; Ripamonti et al. 2008; Thomas \& Zaroubi 2008). A positive feedback would arise if $\mathrm{H}_{2}$ molecule formation were promoted due to the elevated fraction of free electrons from X-ray photoionization, facilitating the cooling and collapse of gas and thus star formation inside minihalos. Alternatively, X-ray heating may have suppressed star formation by increasing the entropy floor in the pre-galactic medium, implying a negative feedback.

Miniquasar feedback may also be expected to have important effects on BH growth. One outstanding question is how the supermassive black holes (SMBHs), observed by the Sloan Digital Sky Survey (SDSS) at $z \sim 6$ with masses of $\sim 10^{9} M_{\odot}$ (Fan et al. 2006), were able to grow within such a short period of time after the big bang (Haiman \& Loeb 2001). To answer this question, some possible scenarios have been suggested, depending on the initial seed mass (Bromm \& Yoshida 2011). One of the main ideas is that the SMBH is born as a stellar-mass $\mathrm{BH}$, the remnant of a Pop III star with a mass close to that of its progenitor, and grows through the accretion of surrounding gas and mergers with BHs that form in neighboring halos (Madau \& Rees 2001; Li et al. 2007; Volonteri \& Rees 2006; Tanaka \& Haiman 2009). However, the photoheating and photoionization from a massive Pop III star is strong enough to significantly suppress a further supply of cold gas onto the $\mathrm{BH}$, creating a serious early bottleneck to growth (Johnson \& Bromm 2007; Pelupessy et al. 2007; Alvarez et al. 2009; Park \& Ricotti 2011).

A competing scenario suggests the direct collapse of primordial gas into an atomic cooling halo in the absence of lowtemperature coolants such as $\mathrm{H}_{2}$, or metals and dust. Due to the lack of low-temperature coolants, the fragmentation of the gas will be avoided, and the ensuing isothermal collapse may result in the direct formation of a $\mathrm{BH}$ without Pop III stellar progenitor. As a result, the mass of the seed $\mathrm{BH}$ is on the order of $10^{4}-10^{5} M_{\odot}$, allowing subsequent growth from a more massive foundation (Bromm \& Loeb 2003; Koushiappas et al. 2004; Wise \& Abel 2008; Regan \& Haehnelt 2009; Schleicher et al. 2010; Shang et al. 2010; Johnson et al. 2011; Latif et al. 2011). In this scenario, negative feedback due to photoheating and radiation pressure may still oppose growth (Johnson et al. 2011). A second goal of this study is to provide an improved understanding of whether a stellar $\mathrm{BH}$ can grow to become an $\mathrm{SMBH}$ in the presence of stellar and $\mathrm{BH}$ feedback.

Here, we present the results of cosmological simulations, starting from the point where Pop III stars are first expected to form, up to the virialization of a primordial galaxy, taking into account both the ionizing radiation emitted by Pop III stars and by miniquasars. We assume that all Pop III stars end their lives as massive BHs without $\mathrm{SN}$, allowing us to investigate solely the feedback from miniquasars and to compare our results with the case where Pop III stars die as PISNe. The outline of this paper is as follows. In Section 2, we describe the numerical methodology for the initial setup, the stellar radiative feedback, and the feedback from miniquasars. In Section 3, we show how the primordial galaxy is assembled with and without $\mathrm{BH}$ feedback. Then, in Section 4, we discuss the properties of the gas as it is falling into the center of the galaxy. We present our conclusions in Section 5.

In the following, all lengths are expressed in physical (i.e., not comoving) units unless explicitly stated otherwise.

\section{NUMERICAL METHODOLOGY}

In this section, we describe the initial setup of the simulations and our methodology for calculating the accretion rate onto $\mathrm{BHs}$, as well as our treatment of radiative feedback from the Pop III progenitor stars, an isolated BH remnant, and from an HMXB. 
Table 1

Simulation Parameters

\begin{tabular}{lcc}
\hline \hline Simulation & BH Feedback & BH Multiplicity \\
\hline BHN & No & $\ldots$ \\
BHS & Yes & BH single (isolated) \\
BHB & Yes & BH binary (HMXB) \\
\hline
\end{tabular}

\subsection{Initial Conditions}

To survey the relevant parameter space, we have carried out three cosmological simulations. As a reference, simulation "BHN," includes stellar radiative feedback from Pop III stars, whereas the subsequent feedback due to $\mathrm{BH}$ accretion is not taken into account. The simulation "BHS" includes both the feedback from Pop III stars and a single isolated BH. Finally, in simulation "BHB," we assume that the Pop III BH remnant has a stellar binary companion, giving rise to an HMXB. We provide a summary of the simulations in Table 1.

For the simulations presented here, we use a parallel version of the combined hydrodynamics and tree $N$-body code GADGET2 (Springel 2005). The code evaluates hydrodynamical forces using the SPH technique. Our simulations are initialized using a snapshot from the earlier simulation of Greif et al. (2010). We start running the simulations at $z \sim 30$, corresponding to the point just before the first Pop III star in the computational box is formed, and terminate them at $z \sim 10$, when the assembly of the first galaxy is expected to be complete. The original simulation was initialized at $z=100$ in a periodic box of linear size of $1 \mathrm{Mpc}$ (comoving), using $\Lambda \mathrm{CDM}$ cosmological parameters with matter density $\Omega_{m}=1-\Omega_{\Lambda}=0.3$, baryon density $\Omega_{b}=0.04$, present-day Hubble expansion rate $H_{0}=70 \mathrm{~km} \mathrm{~s}^{-1} \mathrm{Mpc}^{-1}$, spectral index $n_{\mathrm{s}}=1.0$, and normalization $\sigma_{8}=0.9$, consistent with the WMAP5 measurements (Komatsu et al. 2009). Given that we use a higher value compared to the current measurement of $\sigma_{8}=0.8$ (Komatsu et al. 2011), structure formation may be accelerated in our simulations. However, we compensate to some extent for the lack of large-scale power in our simulations due to the limited box size by taking a value larger than the cosmological one. We also stress that in this paper we focus on individual halo properties which are not sensitive to the variation in the $\sigma_{8}$ parameter.

Using a standard zoom-in technique (see Greif et al. 2010), a preliminary run with $64^{3}$ particles was hierarchically refined to generate initial conditions with high-mass resolution inside the region destined to collapse into the first galaxy. Employing three consecutive levels of refinement, the mass of DM and gas particles in the highest resolution region is $m_{\mathrm{DM}} \sim 33 M_{\odot}$ and $m_{\text {sph }} \sim 5 M_{\odot}$, respectively. The corresponding baryonic mass resolution is $M_{\text {res }} \sim 1.5 N_{\text {neigh }} m_{\text {sph }} \sim 400 M_{\odot}$, where $N_{\text {neigh }} \sim 50$ is the number of neighboring particles within the SPH smoothing kernel (Bate \& Burkert 1997). The Jeans mass in primordial gas, where molecular hydrogen cooling imprints a characteristic density of $n_{\mathrm{H}}=10^{4} \mathrm{~cm}^{-3}$ and a temperature of $200 \mathrm{~K}$ (Bromm et al. 2002), is thus marginally resolved.

\subsection{Chemistry, Heating, and Cooling}

We use the same chemistry and cooling network as in Greif et al. (2010), where all relevant cooling mechanisms, such as $\mathrm{H}$ and $\mathrm{He}$ collisional ionization, excitation and recombination cooling, bremsstrahlung, inverse Compton cooling, and collisional excitation cooling via $\mathrm{H}_{2}$ and $\mathrm{HD}$, are taken into account. For $\mathrm{H}_{2}$ cooling, collisions with protons and electrons are explicitly included, in addition to the usually dominant neutral hydrogen atoms. The code self-consistently solves the rate equations for the abundances of $\mathrm{H}, \mathrm{H}^{+}, \mathrm{H}^{-}, \mathrm{H}_{2}, \mathrm{H}_{2}^{+}, \mathrm{He}, \mathrm{He}^{+}, \mathrm{He}^{++}$, and $e^{-}$, as well as the three deuterium species $\mathrm{D}, \mathrm{D}^{+}$, and $\mathrm{HD}$. We can thus accommodate the non-equilibrium chemical evolution which is ubiquitous in early universe structure formation.

\subsection{Sink Particle Method}

Employing the sink particle algorithm of Johnson \& Bromm (2007), we convert an SPH particle into a collisionless sink particle if its hydrogen number density exceeds a threshold value of $n_{\max }=10^{4} \mathrm{~cm}^{-3}$. When a sink particle forms, gas particles within an accretion radius, $r_{\text {acc }}$, are immediately accreted onto the sink. The position and velocity of a sink particle are estimated every time step based on a new massweighted position and velocity of the accreted gas particles within $r_{\text {acc }}=L_{\text {res }}$. Here, the resolution length of the simulation is defined as

$$
L_{\mathrm{res}}=0.5\left(\frac{M_{\mathrm{res}}}{\rho_{\mathrm{max}}}\right)^{1 / 3} \simeq 1 \mathrm{pc}
$$

where $\rho_{\max } \simeq n_{\max } m_{\mathrm{H}}$.

A sink particle can grow in mass through the further accretion of surrounding gas. The criterion for subsequent accretion is that a neighboring SPH particle approaches the sink to within $r_{\mathrm{acc}}$, which we hold constant throughout the simulation. Note that our simple prescription for sink growth is sufficient for the purpose of providing a marker for the position of a Pop III star and its $\mathrm{BH}$ remnant. When determining the mass growth of the $\mathrm{BH}$ which is responsible for the X-ray feedback, we use a more sophisticated methodology to determine its accretion rate (see Section 2.5).

\subsection{Stellar Radiative Feedback}

After a Pop III star has formed inside its host minihalo, we turn on the radiation field emitted by this star, treated as a point source. We propagate the ionization front (I-front) around it to build up a primordial $\mathrm{H}$ II region, using a well-tested raytracing algorithm (Greif et al. 2009). This scheme solves the I-front equation in a spherical grid by tracking $10^{5}$ rays with 500 logarithmically spaced radial bins around Pop III stars. The hydrodynamical effect is taken into account by self-consistently coupling the ray-tracing module to the chemical and thermal evolution of the gas.

We assume that a $100 M_{\odot}$ Pop III star emits blackbody radiation with an effective temperature $T_{\text {eff }}=10^{4.9} \mathrm{~K}$, and luminosity $L_{*}=10^{6.1} L_{\odot}$ (Bromm et al. 2001b; Schaerer 2002). The corresponding production rates for ionizing photons are: $\dot{N}_{\text {ion, H } / \mathrm{He}_{\mathrm{I}}}=9.1 \times 10^{49} \mathrm{~s}^{-1}$ and $\dot{N}_{\text {ion,He II }}=4.1 \times 10^{48} \mathrm{~s}^{-1}$. The growth of the I-front continues until it reaches its maximum size at the end of the Pop III star's life after $t_{*}=2.7$ Myr. However, not all Pop III stars are endowed with an $\mathrm{H}$ II region because of the computational expense incurred by the ray tracing. We trace the photons only from those stars formed within the Lagrangian volume destined to become the first galaxy at $z \sim 10$, roughly corresponding to a $10 \mathrm{kpc}$ radius from the center of this region.

Finally, we include the transfer of the $\mathrm{H}_{2}$-dissociating Lyman-Werner (LW) photons in the range of 11.2-13.6 eV, isotropically emitted by a Pop III star as a $1 / r^{2}$ field without attenuation of the flux with radius, which propagate far beyond the $\mathrm{H}$ II region. The photo-dissociating rate is $k_{\mathrm{H}_{2}}=1.1 \times 10^{8} F_{\mathrm{LW}}$, where $F_{\mathrm{LW}}$ is the radiation flux integrated over the LW bands (Abel et al. 1997). We compute $F_{\mathrm{LW}}$ in the optically thin limit 
and do not take $\mathrm{H}_{2}$ self-shielding into account (see Greif et al. 2009 , for more details). The neglect of self-shielding is justified due to the low $\mathrm{H}_{2}$ column densities encountered in our simulations, of the order of $\sim 10^{13} \mathrm{~cm}^{-2}$ inside the $\mathrm{BH}$ host galaxy (Draine \& Bertoldi 1996; Wolcott-Green \& Haiman 2011).

\subsection{Black Hole Feedback}

In an important difference from Greif et al. (2010), we here assume that all Pop III stars die via direct collapse into BHs, without any preceding SN explosion. For simplicity, however, we allow only one $\mathrm{BH}$ to produce radiative feedback due to the accretion onto it, taken as the first $\mathrm{BH}$ formed during the simulation. The heat input due to this $\mathrm{BH}$ feedback into the pregalactic region is active for $\sim 350 \mathrm{Myr}$, while the radiation from individual Pop III stars is turned on for only $2.7 \mathrm{Myr}$. We next describe our treatment for estimating the accretion rate onto a $\mathrm{BH}$ and the model for the radiation emitted by an isolated $\mathrm{BH}$ and an HMXB.

\subsubsection{Accretion Rate}

We assume that the $\mathrm{BH}$ is embedded in a pressure-supported primordial gas cloud, steadily accreting from it. The corresponding accretion rate is given by the Bondi \& Hoyle (1944) model, where a homogeneous medium which is at rest at infinity accretes onto a point mass. The Bondi-Hoyle rate can be written as

$$
\dot{M}_{\mathrm{BH}}=\frac{4 \pi\left(G M_{\mathrm{BH}}\right)^{2} \rho_{\mathrm{gas}}}{\left(c_{s}^{2}+v_{\mathrm{BH}}^{2}\right)^{3 / 2}},
$$

where the gas sound speed, $c_{s}$, and gas density, $\rho_{\text {gas }}$, are determined by averaging over the $N_{\text {neigh }} \simeq 50 \mathrm{SPH}$ particles closest to the $\mathrm{BH}$. The relative speed of the $\mathrm{BH}$ with respect to the surrounding gas, $v_{\mathrm{BH}}$, is negligibly small throughout the simulation. Varying $N_{\text {neigh }}$ by an order of magnitude does not affect the accretion rates when they are estimated with Equation (2).

By assuming that $10 \%$ of the rest-mass energy of the accreted matter is released as radiation, we normalize the total luminosity according to $L=\epsilon \dot{M}_{\mathrm{BH}} c^{2}$, where $\epsilon=0.1$ is the radiative efficiency and $c$ the speed of light. We estimate that the accretion rate $\dot{M}_{\mathrm{BH}}$ lies between $10^{-12} M_{\odot} \mathrm{yr}^{-1}$ and $10^{-6} M_{\odot} \mathrm{yr}^{-1}$. The former corresponds to the initial situation, where the $\mathrm{BH}$ is located at the center of an $\mathrm{H}$ II region with a temperature of $\sim 10^{4} \mathrm{~K}$ and hydrogen number density of $\sim 1 \mathrm{~cm}^{-3}$. The upper limit is derived from comparison simulations where stellar and $\mathrm{BH}$ radiative feedback is neglected.

It is important to note that the accretion rate used in this work is an upper limit for three reasons. In reality, when the feedback from radiation pressure is also taken into account, the true rates are likely much smaller than the nominal Bondi-Hoyle value (Milosavljević et al. 2009a, 2009b; Park \& Ricotti 2012). We estimate, assuming a typical accretion rate of $10^{-8} M_{\odot} \mathrm{yr}^{-1}$, that the acceleration due to radiation pressure from Thomson scattering and photoionization in the vicinity of the $\mathrm{BH}$, at $r=1 \mathrm{pc}$, is 30 times larger than the gravitational acceleration, $a_{\text {grav }}=G M_{\mathrm{BH}} / r^{2}$ (e.g., Equation (10) in Johnson et al. 2011). This indicates that radiation pressure, mainly due to photoionization, is important near the $\mathrm{BH}$, acting to limit growth. The other reason is that we have only considered the case of radiatively efficient accretion. If the cooling timescale for the liberation of viscously generated energy is longer than the accretion timescale, then most of the energy is advected inward with the accreting gas instead of being radiated away (Narayan \& Yi

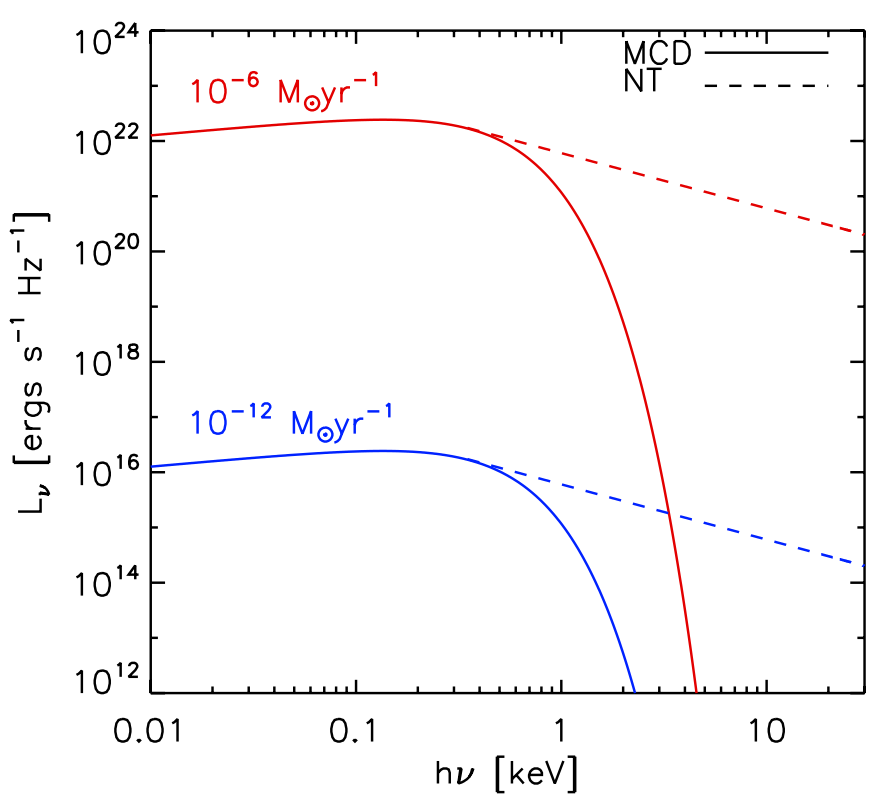

Figure 1. Emission spectra produced by gas accreting onto a $100 M_{\odot} \mathrm{BH}$ with two different accretion rates, $\dot{M}=10^{-6} M_{\odot} \mathrm{yr}^{-1}$ and $\dot{M}=10^{-12} M_{\odot} \mathrm{yr}^{-1}$. "MCD" (solid) shows the continuum spectra from the multi-color disks and "NT" (dashed) denotes a non-thermal component with a power law of $\beta=1$. The combined spectra are normalized to the total luminosity, $L=\epsilon \dot{M} c^{2}$, where $\epsilon=0.1$ is the radiative efficiency, assuming that each component contributes $50 \%$ to the total. Note that in our simulations we ignore the multi-color disk component and instead assume that the radiation emitted by the accreting $\mathrm{BH}$ is characterized by a pure power-law spectrum.

(A color version of this figure is available in the online journal.)

1994, 1995; Blandford \& Begelman 1999, 2004). A fraction of the energy carried by the outflow could ultimately be converted to radiation, but the resulting spectrum would certainly be very different than that of the thin disk assumed here. A third reason is that the vorticity of the turbulent gas has not been properly resolved here. Studying its effect on Bondi accretion, Krumholz et al. (2005) found that even a small amount of vorticity is able to significantly reduce the accretion onto the $\mathrm{BH}$. This suppression could be important in an atomic cooling halo, where the radial, cold flow along the filaments is converted into turbulent motion, thus generating vorticity in the central region of the galaxy.

In addition to the Bondi-Hoyle prescription, there is another way to estimate $\mathrm{BH}$ growth, based on $\dot{M}_{\text {sink }}$ calculated with the method in Section 2.3, where the BH mass grows by accreting all gas particles within $r_{\text {acc }}$. The resulting rate is much higher than the Bondi-Hoyle value, roughly by a factor of 10 . We adopt $\dot{M}_{\mathrm{BH}}$ rather than $\dot{M}_{\text {sink }}$ as an estimator for BH growth, given that the sink accretion radius is about one order of magnitude larger than the Bondi-Hoyle radius, $r_{\mathrm{B}}=\left(\mu m_{\mathrm{H}} G M_{\mathrm{BH}}\right) / k_{\mathrm{B}} T$, where $\mu$ is the mean molecular weight and $k_{\mathrm{B}}$ is the Boltzmann constant, or, $r_{\mathrm{B}} \sim 0.3 \mathrm{pc}\left(M_{\mathrm{BH}} / 10^{2} M_{\odot}\right)\left(T / 10^{2} \mathrm{~K}\right)^{-1}$. Moreover, only a fraction of the infalling gas will likely reach the vicinity of the $\mathrm{BH}$. Furthermore, we can thus track the change in $\mathrm{BH}$ mass in a smooth manner and do not have to contend with discreteness effects owing to the limited mass resolution afforded by SPH.

\subsubsection{Non-thermal Radiation}

In the local universe, the emergent spectra from accreting BHs are typically modeled as a combination of a power-law component, $F_{v} \propto v^{\beta}$, describing non-thermal (NT) synchrotron radiation, and a multi-color disk (MCD), resulting in a soft, thermal continuum (e.g., Mitsuda et al. 1984). In Figure 1, 
we show typical spectra to illustrate this model where a soft thermal component is fitted by an MCD blackbody with $k T_{\text {in }} \sim 150 \mathrm{eV}$ (Miller et al. 2003), normalized assuming two representative accretion rates. Here, $T_{\text {in }}$ is the temperature of the inner disk region which is related to the $\mathrm{BH}$ mass according to $T_{\text {in }} \propto M_{\mathrm{BH}}^{-1 / 4}$. It is currently not known whether accreting BHs at high redshift would behave in a similar manner.

Conservatively, we here assume that there is no significant difference in the $\mathrm{BH}$ emission physics over cosmic time and use the same spectra. However, we ignore the MCD component of the emitted radiation in our simulations. Such a thermal contribution would determine the radiative feedback in the immediate "near zone" from the central $\mathrm{BH}$, but is unimportant on larger scales, where the NT component dominates. We estimate the size of the near zone with a standard Strömgren analysis. Assuming that the ionizing photons originate from the MCD component, and employing a typical accretion luminosity of $\dot{M}_{\mathrm{BH}}=10^{-7} M_{\odot} \mathrm{yr}^{-1}$ and a typical density of $n_{\mathrm{H}}=10 \mathrm{~cm}^{-3}$, we compute an equilibrium I-front radius of $\lesssim 5 \mathrm{pc}$, which is comparable to the spatial resolution of the simulations.

We follow Kuhlen \& Madau (2005) to model the propagation of high-energy photons where the isotropic radiation field, $\propto 1 / r^{2}$, is a function of distance from the $\mathrm{BH}$ only. The coefficient for the ionization rate, $k_{\text {ion }}$, can be written as

$$
k_{\text {ion }}=\int_{v_{\mathrm{NT}}}^{v_{\max }} \frac{F_{\nu} \sigma_{v}}{h v} d v,
$$

where $v_{\mathrm{NT}}=0.2 \mathrm{keV} / h$ is the frequency where the NT component starts to dominate and $v_{\max }=10 \mathrm{keV} / h$ is the upper cutoff frequency. Our choice of energy range, $0.2-10 \mathrm{keV}$, is motivated by the study of Miller et al. (2003), where the spectra of select ultraluminous X-ray sources (ULXs) associated with $100 M_{\odot}$ BHs were fitted with a power law in this same range. For the hydrogen and helium photoionization cross sections, $\sigma_{v}$, we use the standard expressions (e.g., Barkana \& Loeb 2001; Osterbrock \& Ferland 2006).

To consider the secondary ionization effects from energetic electrons released through the absorption of an X-ray photon, we adopt the fitting formulae given by Shull \& van Steenberg (1985; see also Valdés \& Ferrara 2008; Furlanetto \& Stoever 2010). They computed the fractions of the initial electron energy going into secondary ionizations of $\mathrm{HI}$, secondary ionizations of $\mathrm{He} \mathrm{I}$, and into heating the surrounding gas. Secondary ionizations of He II are not important (Shull \& van Steenberg 1985) and are thus not included in the overall energy budget. The effective ionization rates can thus be written as

$$
k_{\text {eff }}\left[\mathrm{HI}_{\mathrm{I}}\right]=\mathrm{k}_{\mathrm{ion}}[\mathrm{H} \mathrm{I}]+\mathrm{k}_{\mathrm{sec}}[\mathrm{H} \mathrm{I}]
$$

where

$$
k_{\mathrm{sec}}[\mathrm{HI}]=\mathrm{f}_{\mathrm{H}}\left(\Gamma_{\mathrm{HI}_{\mathrm{I}}}+\frac{\mathrm{n}_{\mathrm{He}} \mathrm{I}}{\mathrm{n}_{\mathrm{HI}}} \Gamma_{\mathrm{He}}\right) \frac{1}{13.6 \mathrm{eV}}
$$

and

$$
k_{\text {eff }}[\mathrm{He} \mathrm{I}]=\mathrm{k}_{\text {ion }}[\mathrm{He} \mathrm{I}]+\mathrm{k}_{\mathrm{sec}}[\mathrm{He} \mathrm{I}]
$$

where

$$
k_{\mathrm{sec}}[\mathrm{He} \mathrm{I}]=\mathrm{f}_{\mathrm{He}}\left(\Gamma_{\mathrm{He} \text { I }}+\frac{\mathrm{n}_{\mathrm{HI}}}{\mathrm{n}_{\mathrm{He}}} \Gamma_{\mathrm{HI}}\right) \frac{1}{24.6 \mathrm{eV}} .
$$

Here, $\Gamma$, the rate at which the excess energy is released due to the first photoionization of the gas by X-rays, is given by

$$
\Gamma=n_{\mathrm{n}} \int_{v_{\mathrm{NT}}}^{v_{\max }} F_{\nu} \sigma_{v}\left(1-\frac{v_{\min }}{v}\right) d v
$$

where $n_{\mathrm{n}}$ is the number density of the respective unionized species, $v_{\min }$ the ionization threshold frequency, and $h v_{\min }=$ $13.6 \mathrm{eV}, h v_{\min }=24.6 \mathrm{eV}$, and $h v_{\min }=54.4 \mathrm{eV}$ for hydrogen, neutral helium, and singly ionized helium, respectively.

The fractions going into secondary ionizations, $f_{\mathrm{H}}$ and $f_{\mathrm{He}}$, are a function of the hydrogen ionization fraction, $x_{\mathrm{ion}}=n_{\mathrm{H}+} / n_{\mathrm{H}}$, and can be expressed as

$$
\begin{gathered}
f_{\mathrm{H}}=0.3908\left(1-x_{\mathrm{ion}}^{0.4092}\right)^{1.7592} \\
f_{\mathrm{He}}=0.0554\left(1-x_{\mathrm{ion}}^{0.4614}\right)^{1.6660},
\end{gathered}
$$

and the photoheating rates are then $\mathcal{H}=\Gamma\left(1-f_{\mathrm{H} / \mathrm{He}}\right)$.

The flux from the NT component is given by

$$
F_{v}=F_{v_{0}}\left(\frac{v}{v_{0}}\right)^{-\beta}, \quad \beta=1,
$$

in the range of $0.2-10 \mathrm{keV}$, implying a mean hydrogen-ionizing photon energy of $390 \mathrm{eV}$. Here, $F_{v_{0}}$, the flux at $v_{0}=v_{\mathrm{NT}}$, is determined at every time step, based on the normalization

$$
\epsilon \dot{M}_{\mathrm{BH}} c^{2}=\int_{v_{\mathrm{NT}}}^{v_{\max }} L_{\nu} d \nu
$$

where $L_{v}=4 \pi r^{2} F_{v}$ is the specific luminosity. We note that since extinction along the line of sight is not taken into account, the estimated ionization and heating rates are likely upper limits. Recall that the accretion rate, $\dot{M}_{\mathrm{BH}}$, is updated at every time step, thus allowing our algorithm to reflect the changing conditions in the vicinity of the $\mathrm{BH}$.

The resulting photoionization and photoheating rates are a function of distance from the $\mathrm{BH}$, the instantaneous accretion rate, a function of the hydrogen ionization fraction, and can be conveniently written as

$$
k_{\mathrm{ion}}=\dot{K}\left(\frac{r}{\mathrm{pc}}\right)^{-2}\left(\frac{\dot{M}_{\mathrm{BH}}}{10^{-6} M_{\odot} \mathrm{yr}^{-1}}\right),
$$

where

$$
\dot{K}=[1.96,2.48,0.49] \times 10^{-11} \mathrm{~s}^{-1}
$$

and

$$
\mathcal{H}=n_{j} \dot{H}\left(\frac{r}{\mathrm{pc}}\right)^{-2}\left(\frac{\dot{M}_{\mathrm{BH}}}{10^{-6} M_{\odot} \mathrm{yr}^{-1}}\right)\left(1-f_{\mathrm{H} / \mathrm{He}}\right),
$$

where

$$
\dot{H}=[7.81,9.43,1.63] \times 10^{-21} \mathrm{erg} \mathrm{s}^{-1}
$$

for neutral hydrogen, neutral helium, and singly ionized helium, respectively. The corresponding number densities of each species $j$ are given by $n_{j}$.

\subsubsection{HMXB Emission}

Recent improved simulations of Pop III star formation suggest that the primordial gas could fragment into two or more distinct cores, possibly resulting in a massive binary, or higher-multiple stellar system (Turk et al. 2009; Stacy et al. 2010, 2012; Clark et al. 2011a, 2011b; Greif et al. 2011; Smith et al. 2011; Prieto et al. 2011). This picture provides another possible $X$-ray source connected to the first stars, where the primary in a binary system leaves a BH behind at the end of its short life (Mirabel et al. 2011; 
Haiman 2011). The remnant could remain bound to the slightly less massive secondary, where nuclear burning is still going on. The material lost by the secondary in a wind or by Roche lobe overflow will be dumped onto the companion $\mathrm{BH}$, releasing copious X-ray emission. The duration of such an HMXB is limited by the main-sequence lifetime of the donor and is of the order of $10^{7} \mathrm{yr}$.

It has been suggested that there is a correlation between the number of HMXBs ULXs, which can be explained as a subpopulation of the former, and low-metallicity environment (Majid et al. 2004; Dray 2006; Soria 2007; Mapelli et al. 2009; Linden et al. 2010). We can thus expect that the number densities of HMXBs and ULXs at high redshifts, when the universe was chemically pristine, were higher than what is observed locally (Mirabel et al. 2011; Haiman 2011). Such local surveys show that HMXBs and ULXs have luminosities of $L_{X} \sim 10^{38} \mathrm{erg} \mathrm{s}^{-1}$ and $L_{X} \gtrsim 10^{40} \mathrm{erg} \mathrm{s}^{-1}$, respectively, which are close to the Eddington value, or mildly superEddington (Grimm et al. 2003). Consequently, we assume that the luminosity of an HMXB at high redshifts is close to the Eddington limit, $L_{\mathrm{Edd}}=4 \pi G M_{\mathrm{BH}} m_{\mathrm{H}} c / \sigma_{\mathrm{T}}$, where $m_{\mathrm{H}}, \sigma_{\mathrm{T}}$, and $c$ denote the proton mass, Thomson cross section, and the speed of light, respectively. We further assume that the spectrum exhibits the same power-law behavior as in Equation (11). The X-ray luminosity in the HMXB scenario is then determined from

$$
L_{\mathrm{Edd}}=1.38 \times 10^{40} \mathrm{erg} \mathrm{s}^{-1}\left(\frac{M_{\mathrm{BH}}}{100 M_{\odot}}\right)=\int_{\nu_{\mathrm{NT}}}^{\nu_{\max }} L_{\nu} d \nu,
$$

and we assume $M_{\mathrm{BH}}=100 M_{\odot}$.

\section{FIRST GALAXY ASSEMBLY}

In this section, we present the results obtained from our simulations. In Section 3.1, we briefly discuss the properties of the HiI regions around massive Pop III stars in order to check for agreement between our work and previous studies. We proceed in Section 3.2 to derive the star formation rate in the Lagrangian volume of the emerging primordial galaxy. We then discuss in Section 3.3 how this galaxy is assembled, contrasting the simulations of feedback from a single isolated accreting BH (BHS) and from an HMXB (BHB) described in Section 2 with a simulation without BH feedback (BHN).

Note that the BHN, BHS, and BHB simulations end at different redshifts, corresponding to $z \approx 11.6, z \approx 10.4$, and $z \approx 15.8$, respectively. The reason that we terminated the BHN run earlier than the BHS case is mainly due to computational expense, but the galaxy at $z \approx 11.6$ already meets the criterion for first galaxies and the results give us enough information to compare with those of the BHS run. For the BHB case, we have performed the simulation for much longer than any realistic lifetime of HMXBs. For example, the main-sequence lifetime of a donor star of $30 M_{\odot}$, close to the predicted typical Pop III mass (Clark et al. 2011b; Hosokawa et al. 2011; Stacy et al. 2012), would be $\sim 20$ Myr. Thus, our work can be considered to represent the case where at least one $\mathrm{HMXB}$ exists continuously over $150 \mathrm{Myr}$, somewhere in the Lagrangian volume of the emerging protogalaxy.

\subsection{H II Regions around the First Stars}

The first Pop III star to appear in our cosmological box forms at a redshift $z=28$ inside a $5 \times 10^{5} M_{\odot}$ minihalo. Since the Pop III star is assumed to be very massive, with $M_{*} \simeq 100 M_{\odot}$,

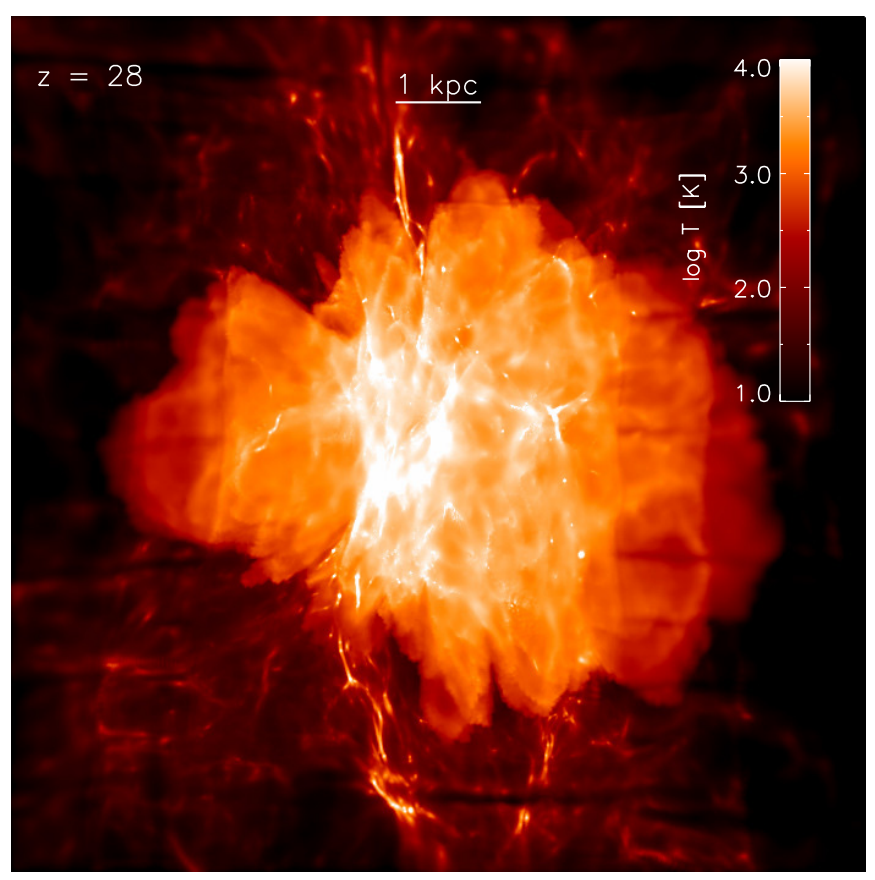

Figure 2. Radiative feedback from the first star. We show the projected temperature distribution, employing a density-squared weighting scheme, of the $\mathrm{H}$ II region around a $100 M_{\odot}$ Pop III star at $z=28$, briefly after its death. The size (see footnote 4 ) of the final $\mathrm{H}$ II region, $\sim 5 \mathrm{kpc}$, greatly exceeds the virial radius of the host halo, $\sim 100 \mathrm{pc}$.

(A color version of this figure is available in the online journal.)

it produces copious amounts of ionizing photons which carve out an extended HiI region into the surrounding IGM. The gas within the $\mathrm{HII}$ region is photoheated to temperatures, $T \sim 3 \times 10^{4} \mathrm{~K}$, in excess of the virial temperature of the minihalo, thus triggering a hydrodynamic outflow. In Figure 2, we show the projected temperature approximately 1 Myr after the radiation from the central star was switched off. The terminal size of the $\mathrm{H}$ II region is about $\sim 5 \mathrm{kpc},{ }^{4}$ such that the I-front extends much farther out than the virial radius of the minihalo, $r_{\mathrm{vir}} \sim 100 \mathrm{pc}$. Note that the anisotropic morphology of the H II region reflects the inhomogeneous density distribution in the neighboring IGM. We describe the physical properties of the photoionized gas in Figure 3, again $\sim 1$ Myr after the Pop III star died. At this time, the gas toward the center has already begun to cool and recombine, leading to a relic $\mathrm{H}$ II region.

The expansion of the I-front begins with a very short lived Rtype (supersonic) phase, followed by a D-type (subsonic) one, where the I-front is trapped behind a hydrodynamical shock wave (e.g., Whalen et al. 2004; Alvarez et al. 2006; Johnson et al. 2007). After $\sim 10^{5} \mathrm{yr}$, the I-front is able to break out, now again supersonically racing ahead of the shock into the low-density IGM. The resulting density profile, together with the location of the shock at $\sim 200 \mathrm{pc}$, and a corresponding velocity of $v_{\mathrm{sh}} \sim 25-35 \mathrm{~km} \mathrm{~s}^{-1}$ are in good agreement with

\footnotetext{
4 A numerical error in the code for the ionization front tracking caused the simulations presented in this work to overestimate the radii of the Pop III H II regions by a factor of $\sim 2$, implying a final $\mathrm{H}$ II region radii of $\sim 5 \mathrm{kpc}$ instead of $\sim 2-3 \mathrm{kpc}$ (compare with, e.g., Greif et al. 2009, their Figure 3). However, this overestimate of the radii does not affect the conclusions of the present work. This is because we are discussing the differences between simulations with and without black hole feedback, which was correctly implemented, and because the radii of the $\mathrm{H}$ II regions were overestimated in a systematic manner by the same factor in all these simulations. In any case, the simulations are consistent with the case including feedback from more massive Pop III stars with larger $\mathrm{H}$ II regions.
} 

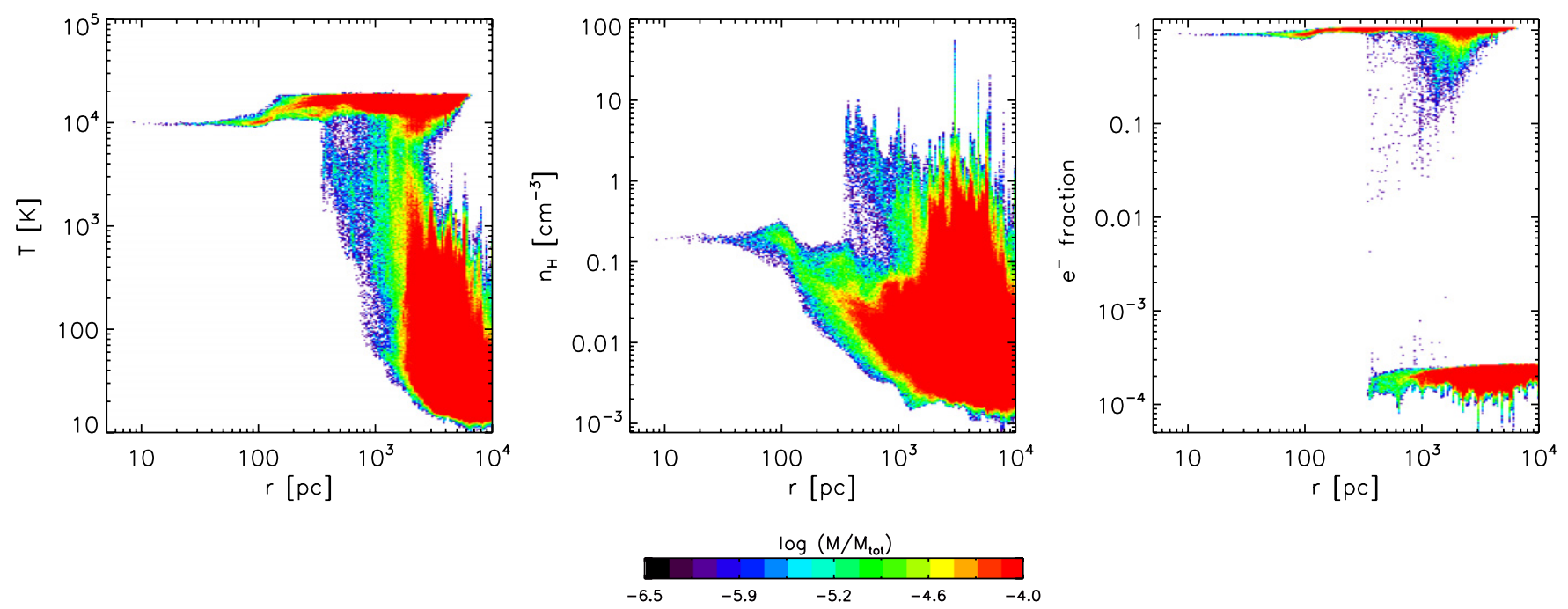

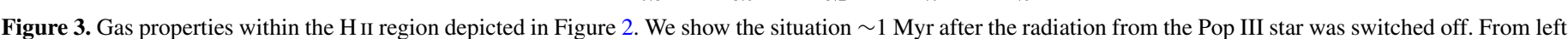

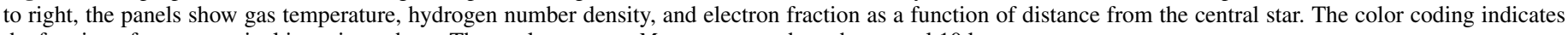
the fraction of mass per pixel in a given phase. The total gas mass, $M_{\mathrm{tot}}$, corresponds to the central $10 \mathrm{kpc}$.

(A color version of this figure is available in the online journal.)

the analytical champagne-flow solution (Shu et al. 2002). We note that contrary to the minihalos of $\sim 10^{6} M_{\odot}$ common at high redshifts, the extent of the shock radius is likely to decrease in rare higher-mass halos, with $\gtrsim 10^{7} M_{\odot}$, due to their deeper potential wells and the effect of infalling gas, possibly trapping the I-front within the host halo, resulting in a compact $\mathrm{H}$ in region (Kitayama et al. 2004; Yoshida et al. 2007a).

At the end of the life of the Pop III star after $~ 2.7 \mathrm{Myr}$, when it leaves a massive $\mathrm{BH}$ remnant behind, the surrounding gas has been evacuated from the minihalo, thus preventing the relic $\mathrm{BH}$ from accreting any cold gas for an extended period of time. After the radiation from the central star shuts down, the relic $\mathrm{H}$ II region starts cooling partly due to adiabatic expansion cooling, and partly due to atomic hydrogen line cooling, facilitated by collisional excitations from the enhanced abundance of free electrons. The elevated electron fraction catalyzes the formation of $\mathrm{H}_{2}$ and $\mathrm{HD}$ molecules in the relic $\mathrm{H}$ II region, thus providing additional molecular cooling (Ricotti et al. 2001; Johnson et al. 2007). Roughly $10 \mathrm{Myr}$ after the radiation from the Pop III star turns off, the temperature decreases from $10^{4} \mathrm{~K}$ to $10^{3} \mathrm{~K}$ and the $\mathrm{H}_{2}$ abundance increases from $10^{-8}$ to $10^{-4}$. While $\mathrm{H}_{2}$ and HD molecules are susceptible to photodissociation from LW radiation produced in neighboring star-forming halos, the relatively short time required for the re-formation of $\mathrm{H}_{2}$ and $\mathrm{HD}$, compared to the average time between the formation of Pop III stars, allows their abundances to remain high.

\subsection{Star Formation}

Figure 4 shows the distance between newly formed Pop III stars and the central BH in the range of redshifts $z=10-20$ from three simulations. A total of $\sim 50$ Pop III stars have been formed in the BHN and BHS cases, accompanied by individual H II regions, according to our criterion that stellar radiative feedback is taken into account only for those stars formed within a $10 \mathrm{kpc}$ radius from the center of the emerging protogalaxy. We find that for over $250 \mathrm{Myr}$ after the seed $\mathrm{BH}$ formed, there has been no further star formation within the host halo in simulation BHS. This is because it takes time for the gas expelled by the $\mathrm{BH}$ progenitor star to be reincorporated into the halo, and the modest feedback from the $\mathrm{BH}$ prevents the gas from cooling.

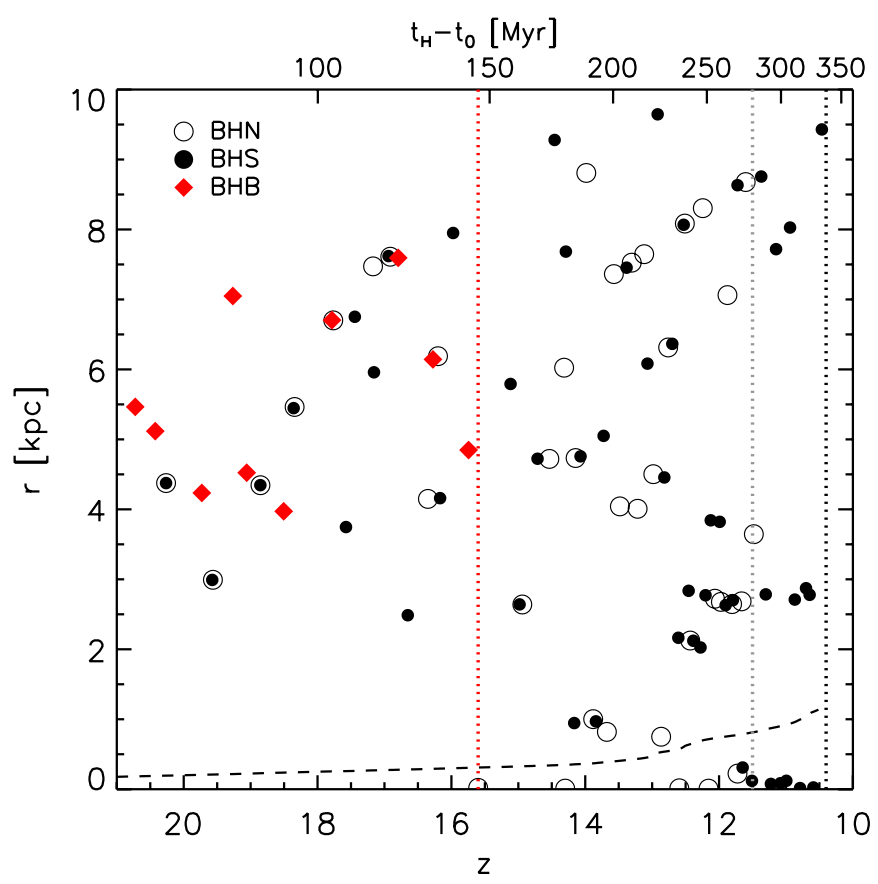

Figure 4. Distances between newly formed Pop III stars that are accompanied by $\mathrm{H}$ II regions and the $\mathrm{BH}$ as a function of redshift from our three simulations. Note that the BHN, BHS, and BHB simulations end at different redshifts, corresponding to $z \approx 11.6, z \approx 10.4$, and $z \approx 15.8$, respectively (indicated by the vertical dotted lines). A total of $\sim 50$ Pop III stars form within $10 \mathrm{kpc}$ around the central $\mathrm{BH}$ in the range of redshifts $z=20-10$ in simulations BHN and BHS. The increase in the number of stars forming at $z \geq 19$ at distances $r \gtrsim 4 \mathrm{kpc}$ from the HMXB in the BHB simulation shows that the HMXB exerts a strong positive feedback on star formation far outside the virial radius of the host galaxy. This is due to the gas collapse into distant minihalos, facilitated via $\mathrm{H}_{2}$ cooling promoted by the strong X-ray emission from the HMXB; locally, on the other hand, star formation is suppressed due to the strong negative feedback from the binary source. We also show the virial radius of the DM halo hosting the active $\mathrm{BH}$ or the HMXB (dashed line). The X-ray photoionization heating from a stellar-mass $\mathrm{BH}$ is also able to quench further star formation in the host halo at all times before the halo enters the atomic cooling phase. The top axis shows the time elapsed since the formation of the $\mathrm{BH}$ at time $t_{0}$.

(A color version of this figure is available in the online journal.) 


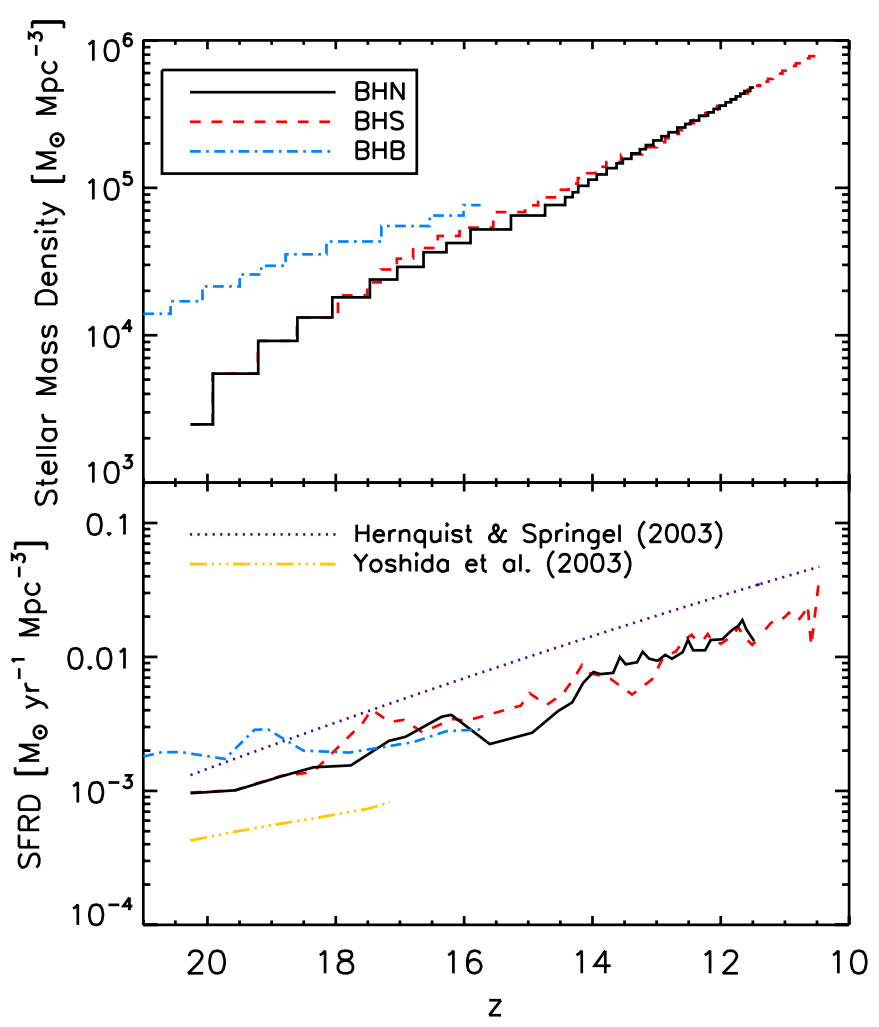

Figure 5. Top panel: comoving stellar-mass density vs. redshift. We compare the results from our three simulations, the BHN comparison case (solid line), the BHS feedback run (dashed line), and the BHB run (dot-dashed line). It is evident that the positive feedback from the BH binary, acting on cosmological scales, has boosted star formation at the highest redshifts. Bottom panel: comoving star formation rate density vs. redshift. The lines refer to the same simulations as above. For comparison, we overplot the analytic fitting formula, derived by Hernquist \& Springel (2003), for higher-mass systems where atomic cooling is active (dotted line), and that of Yoshida et al. (2003) for Pop III star formation in minihalos via $\mathrm{H}_{2}$ cooling (dashed-triple-dotted line).

(A color version of this figure is available in the online journal.)

In simulation $\mathrm{BHN}$, on the other hand, stars inside the host halo continue to form already much earlier, demonstrating that $\mathrm{X}$-ray heating from the accreting $\mathrm{BH}$ in simulation BHS implies a strong local negative feedback.

Note the intense burst of star formation taking place within the virial radius of the protogalactic halo at $z=11.5-10.5$ in the BHS. This starburst is fueled by a massive infall of gas. This gas is able to cool not only via molecular hydrogen cooling but also via atomic hydrogen line cooling. A fraction of the gas mass moving toward the central $\mathrm{BH}$ is thus consumed by star formation rather than being accreted onto the $\mathrm{BH}$.

In Figure 5, we show the evolution of the stellar-mass density and the star formation rate density (SFRD). The comoving stellar-mass density is calculated using the stellar masses that have been formed within the $10 \mathrm{kpc}$ radius from the center of the emerging protogalaxy at a given redshift. For the SFRD, we take the time derivative of the stellar-mass density at the redshift when the Pop III stars formed. For comparison, we overplot SFRDs using analytic fitting formulae (Hernquist \& Springel 2003), for higher-mass halos where atomic hydrogen cooling is dominant, and for minihalos, assuming that one $100 M_{\odot}$ Pop III star forms per system via $\mathrm{H}_{2}$ cooling (Yoshida et al. 2003).

The estimated SFRDs from our simulations lie between the two analytical fits. Evidently, at $z=20$ the SFRD in the BHB run is higher than in the BHN and BHS cases, by a factor of about three. This is a consequence of the positive feedback, where

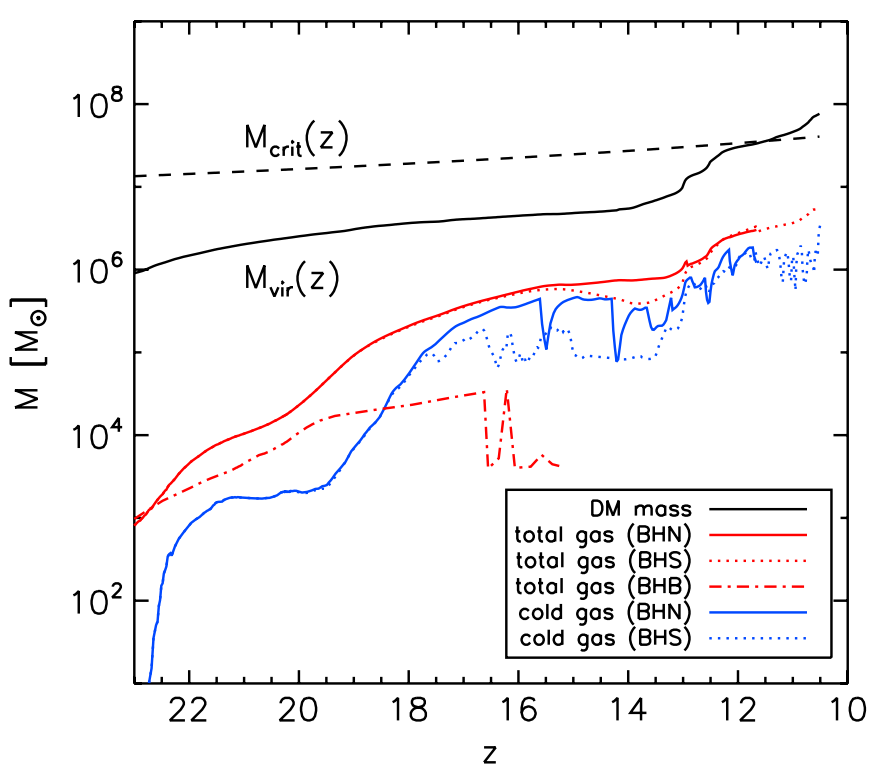

Figure 6. Assembly of the first galaxy. Shown is the redshift evolution of DM virial mass (black solid line) of the halo which will host the first galaxy at $z \sim 10$, as well as of the total gas mass (red) and the cold gas mass (cyan) within the halo for simulations BHN (solid lines), BHS (dotted lines), and BHB (dot-dashed line). The black dashed line represents the critical mass required for the onset of atomic cooling in the halo at a given redshift. There is no cold gas within the halo in simulation $\mathrm{BHB}$ owing to the strong heating from the HMXB.

(A color version of this figure is available in the online journal.)

gas collapse into distant minihalos is facilitated via $\mathrm{H}_{2}$ cooling promoted by the strong X-ray emission from the HMXB. As density fluctuations grow and star formation is enhanced, the local negative radiative feedback from Pop III stars begins to dominate, mitigating the positive HMXB feedback effect. We have not considered cooling due to metals and dust, both produced in SN explosions. At low densities, $n<500 \mathrm{~cm}^{-3}$, and at low metallicities, $Z<10^{-3} Z_{\odot}$; however, $\mathrm{H}_{2}$ cooling is expected to dominate over metal-line cooling (Jappsen et al. 2007, 2009a, 2009b).

\subsection{Mass Growth}

The characteristic property to distinguish the first galaxies from lower mass minihalos is their ability to cool through atomic hydrogen line emission, depending on the virial temperature of the hosting halo with a virial mass $M_{\mathrm{vir}}$ :

$$
T_{\mathrm{vir}} \sim 10^{4} \mathrm{~K}\left(\frac{M_{\mathrm{vir}}}{5 \times 10^{7} M_{\odot}}\right)^{2 / 3}\left(\frac{1+z}{10}\right) .
$$

Above $T_{\text {vir }} \gtrsim 10^{4} \mathrm{~K}$, the gas within the halos is able to cool mainly via atomic hydrogen. In Figure 6, we show the evolution of the virial mass for the most massive halo, which will host the first galaxy at $z \sim 10$, as well as of the total and cold gas masses for the three simulations presented in this work. The virial mass of the DM halo is estimated as the mass within a sphere with average DM density $\rho \sim 200 \rho_{0}(z)$, where $\rho_{0}$ is the mean cosmic density at a given redshift. Cold gas is identified by the condition that the gas temperature is less than half of the halo virial temperature, $T<0.5 T_{\text {vir }}(z)$. We also indicate the critical mass required for the onset of atomic hydrogen cooling at a given redshift.

We find that at $z \gtrsim 18$, the halo is dominated by hot gas, exceeding the amount of cold gas by an order of magnitude. 

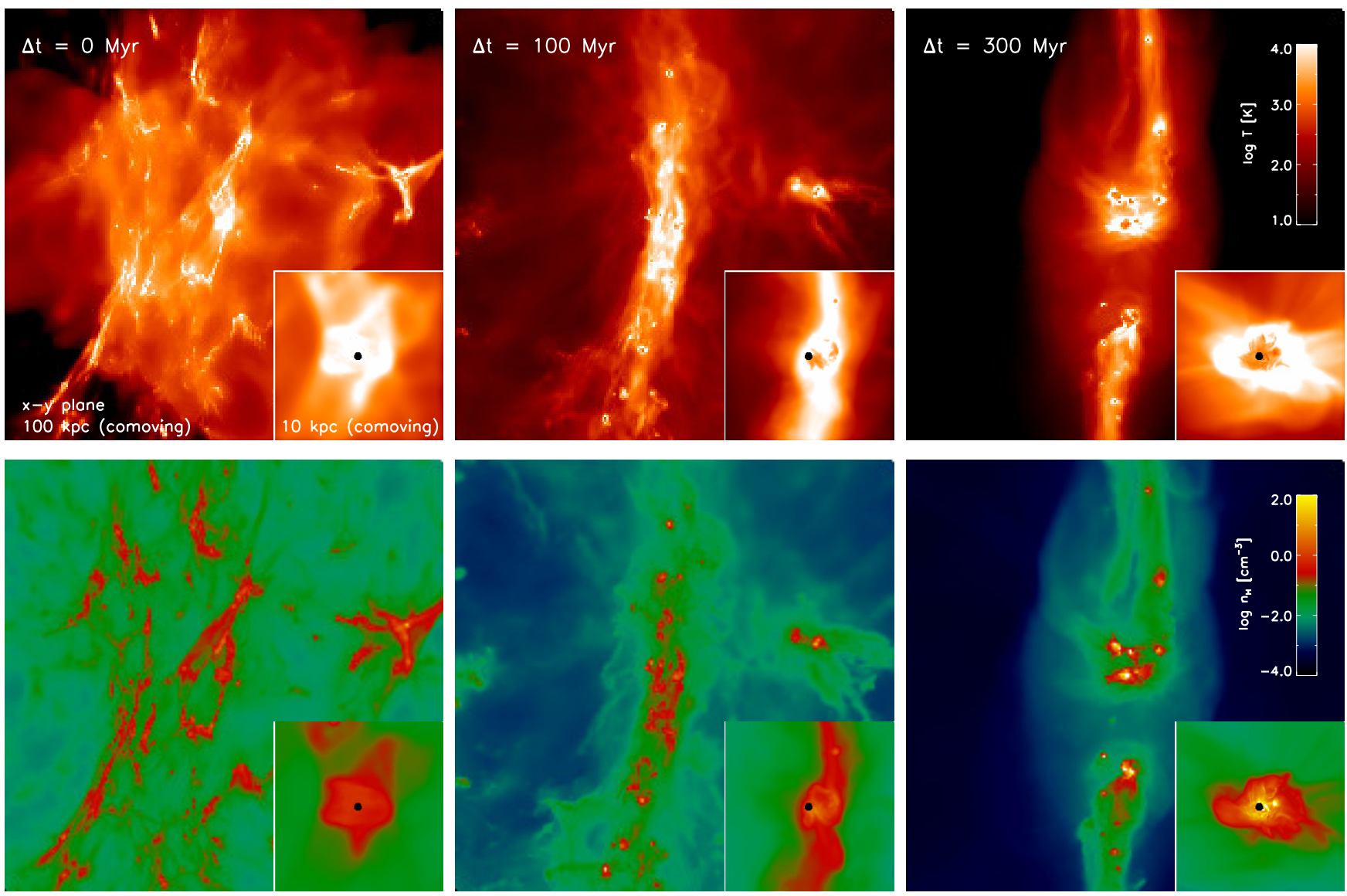

Figure 7. Gas properties in the vicinity of the accreting BH in simulation BHS. Shown are the density-weighted temperature (top row) and the projected hydrogen number density (bottom row) of the gas within cubical slices of linear size $100 \mathrm{kpc}$ (comoving). The small insets provide zooms into the central $10 \mathrm{kpc}$ (comoving). The situation is depicted at three different times, $\Delta t=0,100$, and $300 \mathrm{Myr}$ after the BH has been formed at the center of the minihalo, corresponding to $z=27.9$, 17.7, and 11.0, respectively. The location of the BH is marked by a black circle at the center of each plot.

(A color version of this figure is available in the online journal.)

As time passes on, the cold gas mass increases, eventually accounting for $\gtrsim 80 \%$ of the total gas mass in simulations BHN and BHS. This trend can be understood by the vulnerability of the halo gas to stellar radiative feedback. The corresponding evacuation of gas from the halo is very strong at high redshifts, $z \gtrsim 18$, because the halo potential wells were not yet deep enough to retain photoheated gas. We note that the sharp dips in the amount of cold gas are due to star formation inside the halo itself (at $z \lesssim 12$ ), as well as in neighboring halos that are sufficiently close, within $\lesssim 5 \mathrm{kpc}$. The ionizing feedback from the accreting $\mathrm{BH}$ starts operating at $z<18$, above which the effect is too weak as a result of the initially very low $\mathrm{BH}$ accretion rates.

While the total amount of gas is not sensitive to the $\mathrm{BH}$ feedback, the reduction in cold gas mass by a factor of $\sim 5$ indicates that the additional heating from this feedback, on top of the stellar feedback, has a significant impact on the gas in the center of the forming galaxy. As the halo grows further via smooth accretion and mergers with minihalos, however, at $z \sim 13$, both the total gas mass and mass of cold gas are no longer sensitive to the $\mathrm{BH}$ radiative feedback. At $z \sim 11.5$, the condition for an atomic cooling halo is satisfied.

For simulation BHB, the heating from the HMXB is so strong that all gas particles have temperatures $T>0.5 T_{\mathrm{vir}}(z)$, over the entire range of simulated redshifts $z \gtrsim 15$, and the total gas mass is reduced by an order of magnitude by photoevaporation, as is clearly evident in the dot-dashed lines shown in Figure 6. This result would imply that if an HMXB existed within a minihalo at high redshifts, it would take significantly longer for the halo to reassemble the lost gas and to eventually evolve into a primordial galaxy.

In Figure 7, we show the evolving conditions of the gas in the vicinity of the accreting BH in simulation BHS, while the first galaxy is assembled. Initially, the BH is located at the center of the $\mathrm{H}$ II region produced by the progenitor star. As filamentary structure develops, gas infall and mergers with minihalos frequently occur along the filaments (see the middle panel of Figure 7). Cold accretion along the filaments, which are dense enough to allow molecule re-formation, in turn leading to enhanced cooling, efficiently delivers cold gas into the center of the halo. Roughly 300 Myr after the seed BH formed, the conditions for the onset of atomic hydrogen cooling are met. The virial radius of the halo at this time is $r_{\mathrm{vir}} \sim 1 \mathrm{kpc}$.

\subsection{Black Hole Growth}

Figure 8 shows the evolution of the accretion rate onto the $\mathrm{BH}$, the temperature and density of the neighboring gas, as well as the BH mass for simulations BHN and BHS. For the temperature and density, we average over the 50 closest SPH particles. The BH remnant, originating in the death of a Pop III star, initially resides within a medium of high temperature, $\sim 10^{4} \mathrm{~K}$, and very low density, $n_{\mathrm{H}} \sim 0.1 \mathrm{~cm}^{-3}$, preventing 


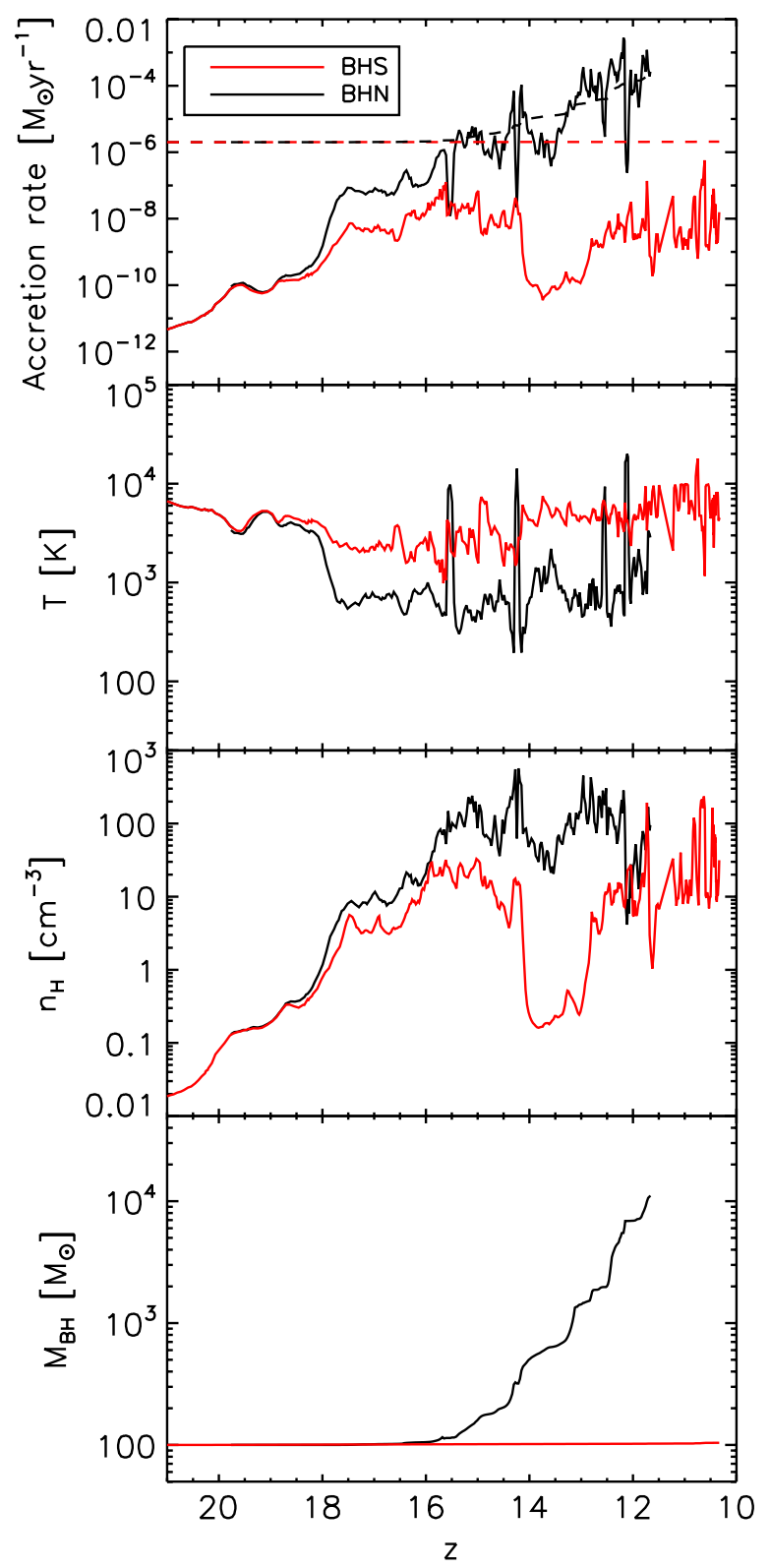

Figure 8. BH growth with and without feedback. Shown are the redshift evolution of the $\mathrm{BH}$ accretion rate, the temperature and density of the gas in the immediate vicinity of the $\mathrm{BH}$, as well as the resulting $\mathrm{BH}$ masses for simulations BHN (black) and BHS (red). In the top panel, we also indicate the corresponding Eddington-limited accretion rates for the two cases (dashed lines).

(A color version of this figure is available in the online journal.)

the gas from cooling and hence accreting onto the $\mathrm{BH}$. At this stage, the accretion rate is only $\dot{M}_{\mathrm{BH}} \sim 10^{-11} M_{\odot} \mathrm{yr}^{-1}$, and the corresponding accretion luminosity, assuming a $10 \%$ radiative efficiency, is $L_{\mathrm{acc}}=\epsilon \dot{M}_{\mathrm{BH}} c^{2} \sim 5.7 \times 10^{33} \mathrm{erg} \mathrm{s}^{-1}$. This value is seven orders of magnitude below the Eddington luminosity, and $\mathrm{BH}$ growth and feedback are negligible in the beginning. As the potential well of the host halo becomes deeper, however, the amount of infalling gas increases with time, boosting the accretion rate, especially at $z \sim 17-19$, as can be seen in Figure 8. Consequently, the accretion luminosity also becomes large enough to influence the surrounding gas, keeping it at a temperature that is an order of magnitude higher than in the BHN comparison simulation.
The accretion rate in the BHN simulation is already comparable to the Eddington value, depicted as dashed lines in Figure 8 (top panel), at $z \sim 15.5$, while it is still an order of magnitude lower in the BHS case. Occasionally, star formation takes place very close to the BH, e.g., $\sim 1 \mathrm{kpc}$ away at $z \sim 14$ (see Figure 4). The radiative feedback from this event acts to compound the heating effect from the $\mathrm{BH}$ accretion, thus rendering the removal of gas out of the shallow potential well more effective. Such gas evacuation at $z \sim 14$ due to both feedback effects is clearly seen in Figure 6.

The combined stellar and $\mathrm{BH}$ radiative feedback results in an accretion rate that is on average four orders of magnitude below the Eddington value at $z=14-13$. Even $300 \mathrm{Myr}$ after the formation of the $\mathrm{BH}$, its mass has increased by only $1.5 \%$ in the BHS simulation, whereas there is a two orders of magnitude growth in the BHN case. This indicates that the feedback from a stellar-mass BH is sufficiently strong to prevent significant growth, suggesting a very important constraint on SMBH formation scenarios. We infer that the radiative feedback from an accreting $\mathrm{BH}$ might be partly responsible for the low density of quasars at redshifts $z \sim 6$, by suppressing early $\mathrm{BH}$ growth.

\section{PROTOGALACTIC GAS PROPERTIES}

In this section, we discuss the properties of the gas as it falls into the center of the emerging galaxy at $z \sim 10$. We compare the situation for our different assumptions on the feedback acting during the preceding assembly, including a discussion of the complementary PISN case. This allows us to constrain the initial conditions for the subsequent starburst inside the first galaxies, a crucial input for predicting their observational signature.

\subsection{Isolated Black Hole Case}

In Figure 9, we compare the temperature, electron fraction, $\mathrm{H}_{2}$ fraction, and HD fraction of the primordial gas within the virial radius of the first galaxy halo as a function of number density for simulations BHN and BHS. We see that at low number densities, $n_{\mathrm{H}}=10^{-3}-1 \mathrm{~cm}^{-3}$, corresponding to the outskirts of the host halo, for both the BHN and BHS simulations the gas temperature is high, $T \sim 10^{3}-10^{4} \mathrm{~K}$, and the electron fraction is enhanced, $f_{e^{-}}=10^{-2}$, due to the accumulated relic $\mathrm{H}$ II regions produced by previous star formation inside and outside of the halo and due to heating in accretion shocks.

In the BHS run, additional heating from the $\mathrm{BH}$ allows the gas within the halo to maintain a relatively high temperature over the entire range of densities. There is a substantial difference seen in the electron fraction: while varying over the large range of $f_{e^{-}}=10^{-6}-1$ in the BHN simulation, in the BHS case most gas within the virial radius has electron fractions above $\gtrsim 10^{-2}$, and there is no gas present with fractions of $<10^{-4}$. In response to the substantially increased electron fraction, the formation of $\mathrm{H}_{2}$ and $\mathrm{HD}$ is catalyzed, leading to molecule abundances that are higher by two orders of magnitude compared to the BHN run.

In the absence of feedback from a $\mathrm{BH}$ in simulation $\mathrm{BHN}$, the molecular hydrogen fraction converges to the well-known asymptotic value of $f_{\mathrm{H}_{2}} \sim 10^{-3}$. This behavior reflects a freeze-out process (Oh \& Haiman 2002), where the molecular hydrogen abundance no longer evolves once the recombination and cooling timescales become less than the $\mathrm{H}_{2}$ formation and dissociation timescales, $t_{\text {rec }}, t_{\text {cool }} \ll t_{\text {form }}, t_{\text {diss }}$. Here, the recombination time is $t_{\mathrm{rec}} \sim 1 /\left(\alpha_{B} n f_{e^{-}}\right)$, and the $\mathrm{H}_{2}$ formation 

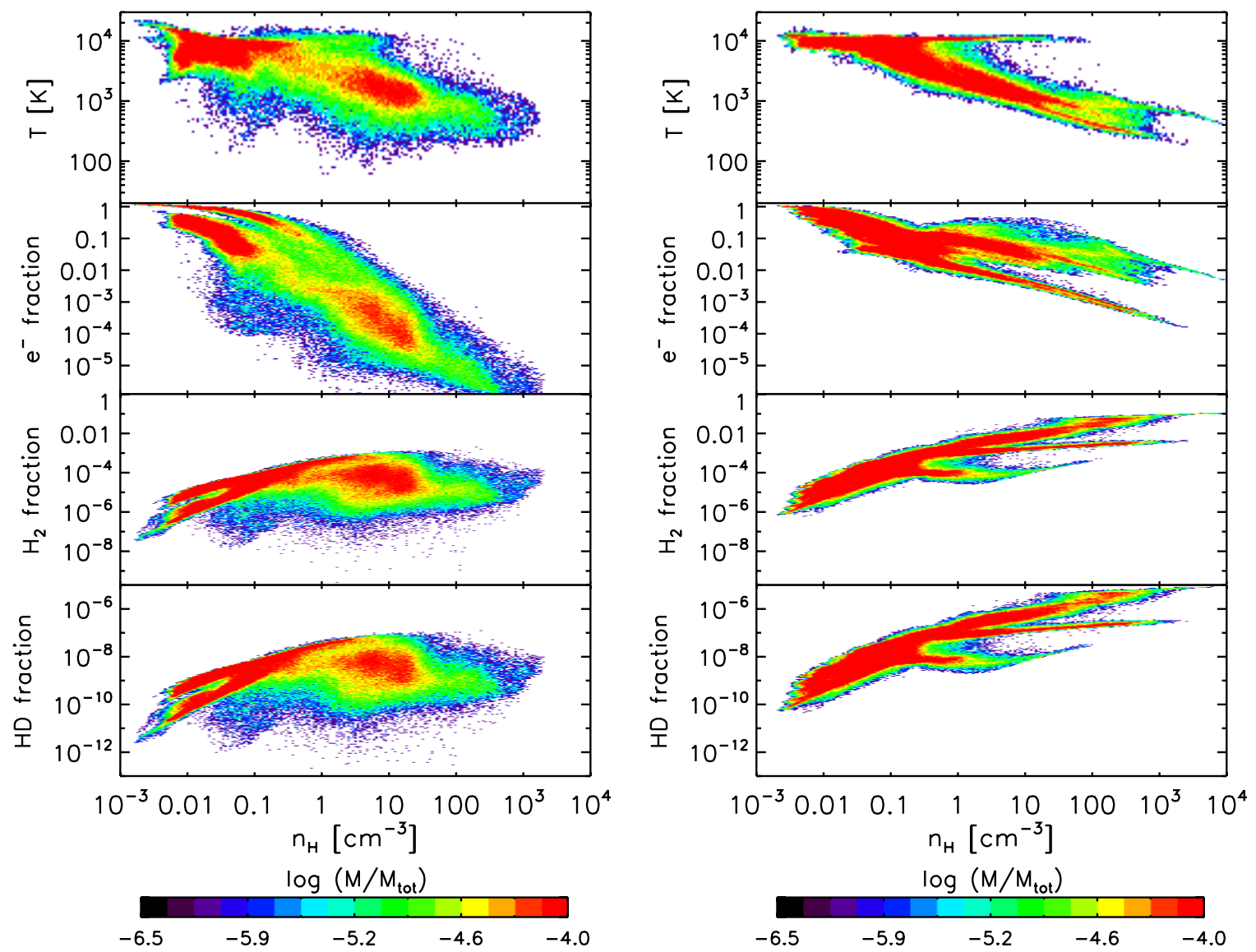

Figure 9. Gas properties within the virial radius of the first galaxy halo. We compare the situation in simulations BHN at $z=11.6$ (left) and BHS at $z=10.4$ (right), corresponding, respectively, to the end of each of the two simulations. From top to bottom, each panel shows the gas temperature, electron fraction, $\mathrm{H}_{2}$ abundance, and $\mathrm{HD}$ abundance, respectively. The feedback from the isolated BH significantly modifies the thermodynamic properties of the central gas. The color coding in these diagrams indicates the fraction of mass in a given phase.

(A color version of this figure is available in the online journal.)

and dissociation timescales are $t_{\text {form }} \sim n_{\mathrm{H}_{2}} / \dot{n}_{\mathrm{H}_{2}, \text { form }}$ and $t_{\mathrm{diss}} \sim$ $n_{\mathrm{H}_{2}} / \dot{n}_{\mathrm{H}_{2} \text {, diss }}$, respectively.

To illustrate the basic difference between the BHN and BHS simulations, we calculate a simple one-zone model, using the same chemistry module as in the simulations, but focusing on a single, representative fluid element as it falls into the center of the emerging galaxy. In the model, the radiation from the $\mathrm{BH}$ is imposed by a $1 / r^{2}$ radiation field, adopting a typical accretion rate of $10^{-8} M_{\odot} \mathrm{yr}^{-1}$, and we assume that the fluid element has a fixed density of $n=10 \mathrm{~cm}^{-3}$, placed $r=100 \mathrm{pc}$ away from the $\mathrm{BH}$ at $z=11.6$. Our fluid element is initially hot, $T=10^{4} \mathrm{~K}$, and highly ionized, typical for a location inside a relic $\mathrm{H}$ II region. The results of the one-zone model are shown in the top panel of Figure 10. To guide the interpretation of these results, we also show, in the bottom panel of Figure 10, the cooling time and the timescales for formation and dissociation of molecular hydrogen, and for the evolution of the electron fraction, $t_{\mathrm{e}} \equiv n_{e} / \dot{n}_{\mathrm{e}}$.

In the case without $\mathrm{BH}$ feedback (solid lines), the $\mathrm{H}_{2}$ formation and dissociation timescales are significantly longer than both the timescales for the evolution of the electron fraction and the cooling rate once the temperature has dropped below a few $10^{3} \mathrm{~K}$, establishing the freeze-out value of $f_{\mathrm{H}_{2}} \sim 10^{-3}$. In the presence of $\mathrm{BH}$ feedback (dashed lines), on the other hand, the additional ionization from the $\mathrm{BH} \mathrm{X}$-ray emission significantly increases the fraction of free electrons at low temperatures. The enhanced electron fraction promotes the formation of $\mathrm{H}^{-}$, and this, at low temperatures, in turn implies an increase in the $\mathrm{H}_{2}$ fraction. We note that similarly high $\mathrm{H}_{2}$ fractions have been found in the related but different context of the non-equilibrium chemistry and cooling of primordial gas behind structure formation shocks under external irradiation (Shapiro \& Kang 1987).

The overall distribution of the HD abundance is similar to that of $\mathrm{H}_{2}$. In the BHS run, the HD fraction increases to $f_{\mathrm{HD}} \sim 10^{-6}$, which exceeds the critical level needed for efficient cooling to the cosmic microwave background (CMB) temperature in local thermodynamic equilibrium, $f_{\mathrm{HD} \text {, crit }} \sim 10^{-8}$, by two orders of magnitude (Johnson \& Bromm 2006), as can be seen in Figure 9. Nevertheless, HD cooling does not succeed in tying the temperature to the $\mathrm{CMB}$ because of the continuous heating from the $\mathrm{BH}$.

We plot the properties of the gas in simulation BHS in Figure 11, but this time as a function of distance from the central $\mathrm{BH}$. The most noticeable feature here is the presence of the two distinct branches toward the center $r<100 \mathrm{pc}$ which is explained by the $\mathrm{BH}$ feedback. To better understand the $\mathrm{BH}$ feedback effect, in Figure 12 we follow the evolution of one representative SPH particle for $\sim 60 \mathrm{Myr}$. Initially, the tracked particle is located at $100 \mathrm{pc}$ away from the central $\mathrm{BH}$, where $\mathrm{BH}$ feedback is negligible. The sudden jumps in both temperature and electron fraction indicate a number of star formation events 


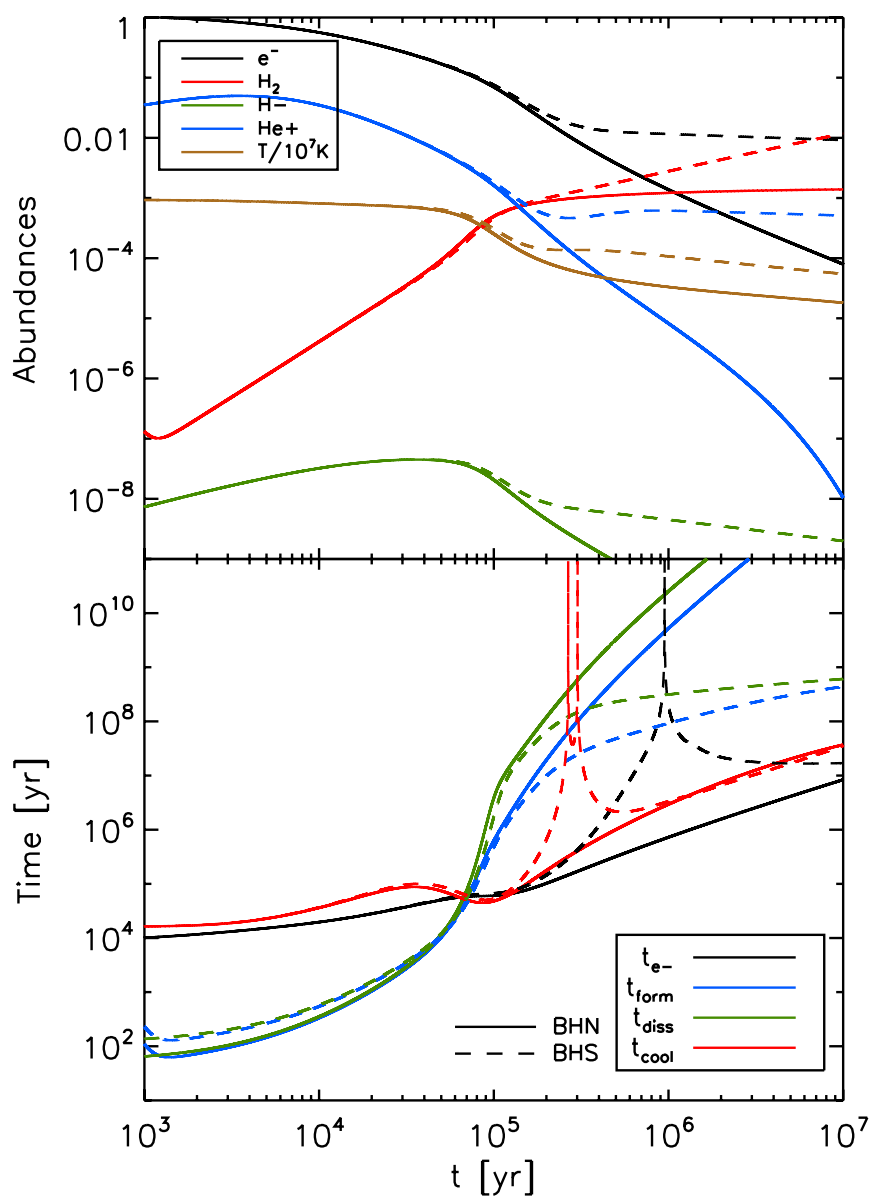

Figure 10. Chemical evolution of the central gas cloud. We calculate an idealized one-zone model, tracing the history of a representative fluid element in the center of the emerging galaxy, starting from $T>10^{4} \mathrm{~K}$ and subsequently cooling down. We employ the same chemistry module as used in the three-dimensional simulations. Top panel: evolution of the particle abundances. Bottom panel: characteristic timescales. In the absence of feedback from a BH (simulation BHN; solid curves), the $\mathrm{H}_{2}$ abundance converges to the asymptotic value of $f_{\mathrm{H}_{2}} \sim 10^{-3}$, reached when both the timescale for the evolution of electron abundance and the cooling time become shorter than the $\mathrm{H}_{2}$ formation and dissociation timescales (Oh \& Haiman 2002). With BH feedback present (BHS; dashed curves), the electron fraction is enhanced due to the X-ray photoionization, and the $\mathrm{H}_{2}$ fraction is enhanced, reaching up to $\gtrsim 10^{-2}$. We note that the additional photoheating and photoionization due to the radiation from a $\mathrm{BH}$ increase the cooling time and the timescale for the evolution of the electron fraction, leading to the peaks clearly seen in the bottom panel.

(A color version of this figure is available in the online journal.)

inside and outside of the halo. Soon after such events, the gas begins to cool back to a few $\sim 10^{3} \mathrm{~K}$, as is typical for relic $\mathrm{H}_{\text {II }}$ regions.

As the gas particle moves within $100 \mathrm{pc}$ from the $\mathrm{BH}$, however, the continuous heating from the accreting $\mathrm{BH}$ starts to operate, resulting in temperatures of $\sim 10^{3} \mathrm{~K}$ and an electron fraction of $f_{e^{-}} \sim 0.1$. As a consequence, $\mathrm{H}_{2}$ molecule formation is catalyzed, leading to fractions of $f_{\mathrm{H}_{2}} \gtrsim 10^{-2}$. Finally, when the gas particle is located too close to the $\mathrm{BH}, r \lesssim 10 \mathrm{pc}$, the strong X-ray flux heats up the gas again to $\sim 10^{4} \mathrm{~K}$, leading to the dissociation of molecules and producing the two distinct branches within the central 100 pc, clearly seen in Figure 11. We point out that the dense clumps around $700-800 \mathrm{pc}$ from the BH in Figure 11 correspond to the middle branch in the $\mathrm{H}_{2}$ and HD molecule fractions, evident in the right panel of Figure 9.

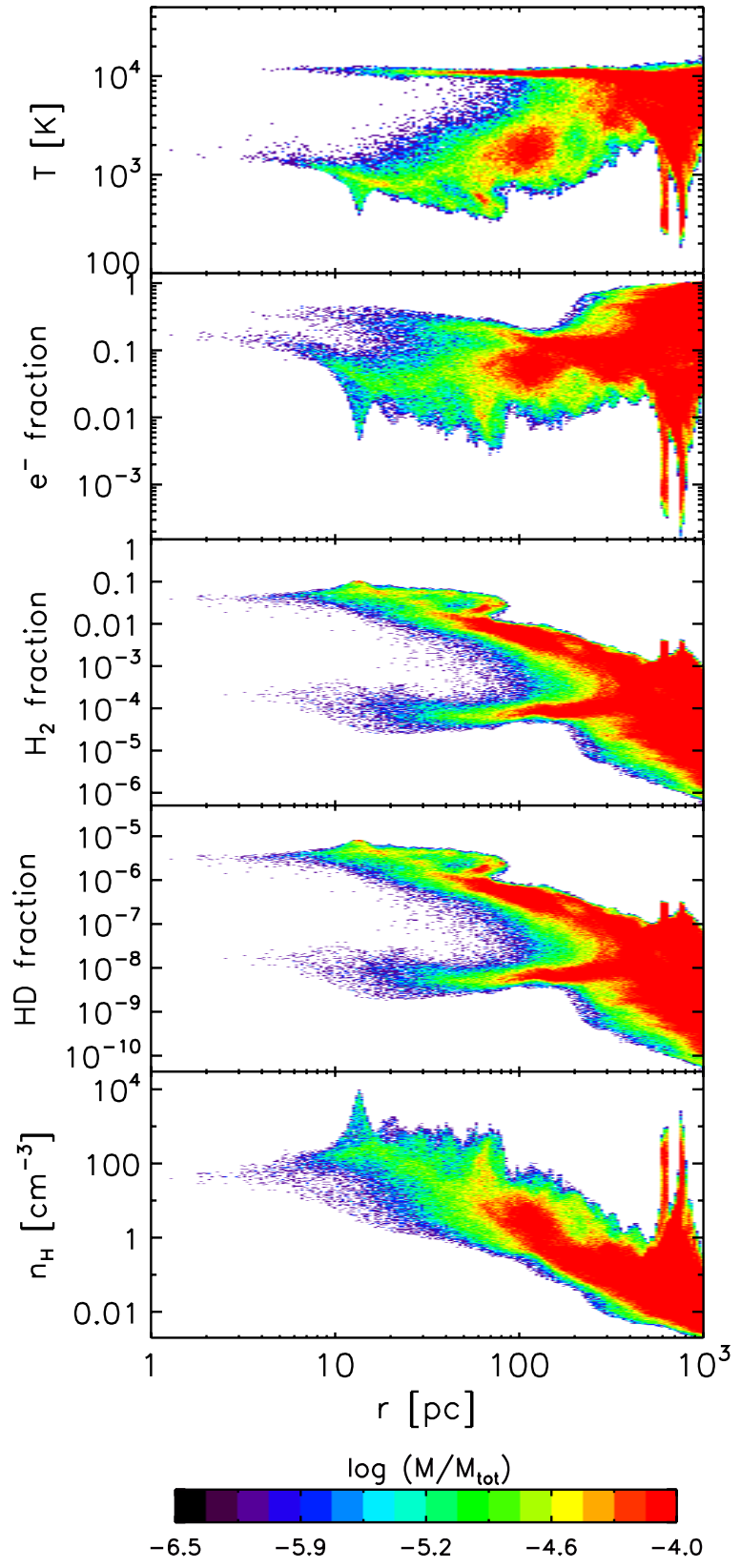

Figure 11. Gas properties in the center of the emerging first galaxy. We show temperature, chemical abundances, and number density vs. radial distance from the galactic center for the same simulation BHS at $z=10.4$ as in the right-hand panel of Figure 9. The radiative feedback from the accreting BH is responsible for the presence of two distinct branches within the central $100 \mathrm{pc}$.

(A color version of this figure is available in the online journal.)

\subsection{Black Hole Binary Case}

In simulation $\mathrm{BHB}$, where the continuous feedback from an HMXB is included, a dramatic change is seen in the gas properties surrounding the central HMXB. Figure 13 shows the comparison between simulations BHN and BHB at $z=15$, plotted in the same fashion as in Figure 11, but now extending to $30 \mathrm{kpc}$ from the HMXB to illustrate the effect of feedback not only on the gas within the host halo but also on the distant IGM. We carefully select the snapshots to guarantee that we are seeing the feedback effect from the HMXB without confusion from Pop III stellar radiation. In the BHB run, the gas temperature within the central $1 \mathrm{kpc}$ reaches $\sim 10^{4} \mathrm{~K}$, or even higher. The $\mathrm{H}_{2}$ and $\mathrm{HD}$ 


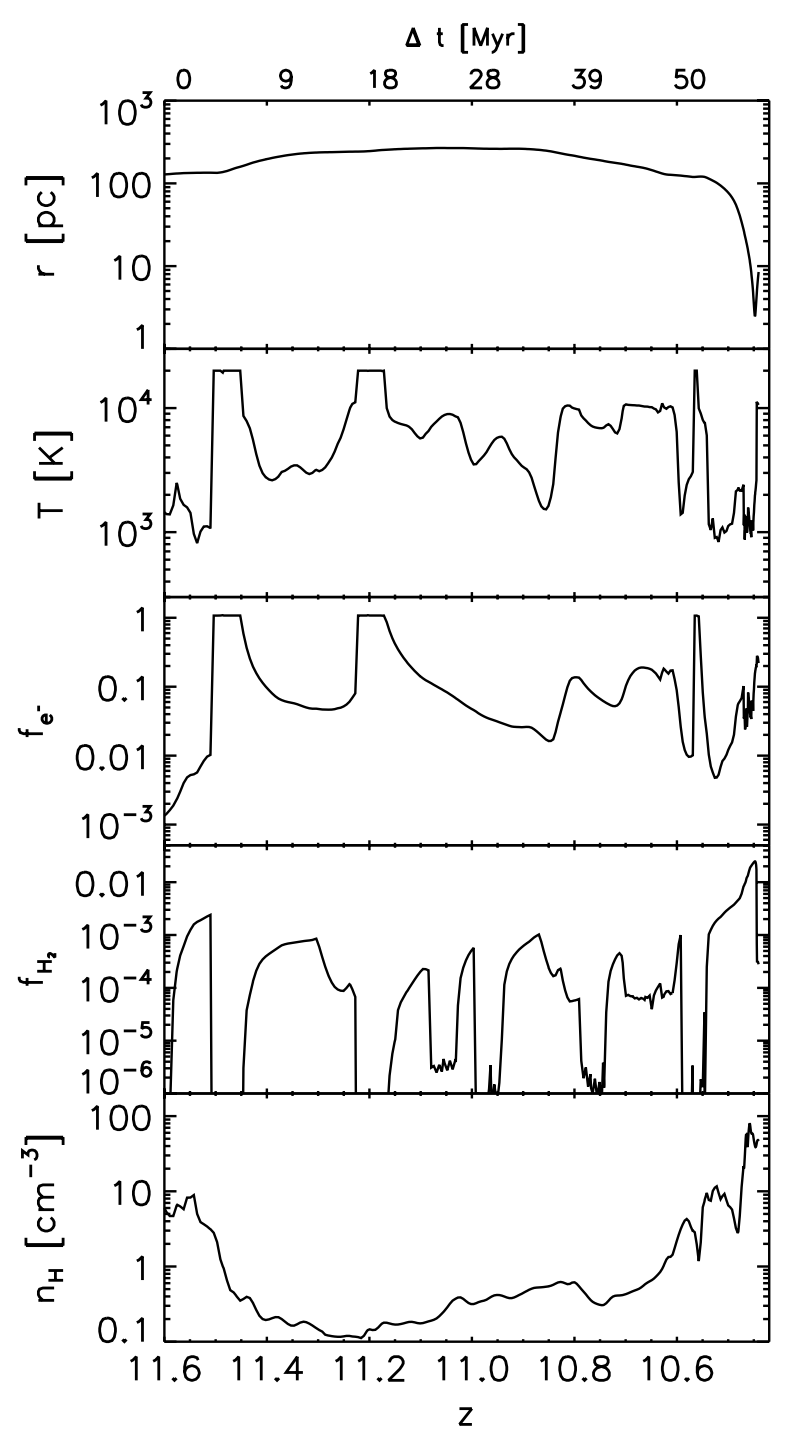

Figure 12. History of a representative SPH particle in the vicinity of the central $\mathrm{BH}$. We follow the evolution for $60 \mathrm{Myr}$, starting at a distance of $100 \mathrm{pc}$ away from the $\mathrm{BH}$, all the way to the center of the emerging first galaxy halo. The spikes in the temperature and electron fraction are due to individual star formation events occurring inside or outside of the growing halo. As the gas particle moves to the center of the halo, feedback from the accreting $\mathrm{BH}$ begins to operate, promoting $\mathrm{H}_{2}$ and $\mathrm{HD}$ molecule formation. Very close to the $\mathrm{BH}$, however, the heating is sufficiently strong to destroy the molecules, generating the two distinct branches for $r \lesssim 50 \mathrm{pc}$ seen in Figure 11 .

molecule abundances are consequently suppressed via effective collisional dissociation, leading to values of $f_{\mathrm{H}_{2}} \sim 10^{-6}$ and $f_{\mathrm{HD}} \sim 10^{-11}$, respectively. The mean density of the gas in the central $100 \mathrm{pc}$ is $n_{\mathrm{H}} \sim 0.01 \mathrm{~cm}^{-3}$ which is four orders of magnitude smaller than that in the BHN run, showing the strong photoevaporation due to HMXB radiative feedback.

In both simulations, part of the IGM outside a $1 \mathrm{kpc}$ radius is heated and ionized by an I-front which had been generated 6.5 Myr ago by Pop III stars formed $\sim 5 \mathrm{kpc}$ away from the center of the halo hosting the HMXB. The HMXB feedback effect on the IGM is most clearly evident in gas which has never experienced any stellar radiation feedback, as the latter could masquerade as the former. The distant IGM in the BHN simulation is in a cold and neutral state, while in the BHB run, the IGM unaffected by any stellar radiative feedback is in a warm and partially ionized phase because of the pervasive effect of the strong HMXB emission. These are favorable conditions for
$\mathrm{H}_{2}$ and $\mathrm{HD}$ molecule formation, promoting their abundance by an order of magnitude over the BHN case.

To more clearly distinguish the HMXB feedback on the first galaxy and on the distant IGM, we compare in Figure 14 the volume-averaged temperature, electron fraction, and $\mathrm{H}_{2}$ fraction for simulations BHN and BHB inside and outside of a $15 \mathrm{kpc}$ radius, beyond which our algorithm does not trigger the buildup of H II regions around newly formed Pop III stars. Hence, any variations in gas properties in the far zone are caused by the HMXB feedback alone. Here, we adopt a $15 \mathrm{kpc}$ cutoff radius rather than $10 \mathrm{kpc}$, the region inside which stars are endowed with an $\mathrm{H}$ II region (see Section 2.4), because an ionized region generated by a Pop III star formed near $r \sim 10 \mathrm{kpc}$ from the center of the emerging galaxy will extend out to $\sim 15 \mathrm{kpc}$. The corresponding X-ray heating increases the volume-averaged temperature within $15 \mathrm{kpc}$ by only a factor of three, while the temperature in the distant IGM is enhanced by two orders of magnitude. The difference in the near zone is relatively small because the gas within the $15 \mathrm{kpc}$ radius is affected by both the feedback from Pop III stars and from the HMXB, indicating that the overall gas properties are dominated by the local stellar feedback. In the absence of any local stellar feedback, as is the case in the region beyond the $15 \mathrm{kpc}$ radius, on the other hand, the IGM is substantially pre-heated and partially ionized when HMXB feedback is present. These conditions facilitate molecule formation, enhancing abundances by an order of magnitude. Our results are in good agreement with the study of Kuhlen \& Madau (2005), given that the X-ray flux in our simulation BHB is comparable to that for their power-law case.

We assume that the HMXB source has been turned on for $\sim 150 \mathrm{Myr}$, which is much longer than any realistic lifetimes. For example, the main-sequence lifetime of a donor star of $30 M_{\odot}$, close to the predicted typical Pop III mass (Clark et al. 2011b; Hosokawa et al. 2011; Stacy et al. 2012), would be $\sim 20$ Myr. Thus, our work can be considered to represent the case where at least one HMXB exists continuously over $150 \mathrm{Myr}$, somewhere in the Lagrangian volume of the emerging protogalaxy. A preheated IGM would provide us with a promising observational window into the early universe. Under these conditions, the IGM would be sufficiently hot to be decoupled from the CMB temperature, $T_{\mathrm{CMB}}=2.73 \mathrm{~K}(1+z)$, but not hot enough to be ionized, leaving the IGM substantially neutral. Such a preheated, neutral IGM is likely detectable in the emission of redshifted $21 \mathrm{~cm}$ radiation, which is produced by the spin-flip transition between the singlet and triplet hyperfine-structure levels of neutral hydrogen. In principle, future $21 \mathrm{~cm}$ observations could provide constraints on the character of early feedback processes, and in particular on the importance of a contribution from Pop III miniquasars (see, e.g., Furlanetto et al. 2006).

\subsection{Comparison to SN Feedback}

In the previous section, we discussed the situation established under radiative feedback from an accreting isolated $\mathrm{BH}$ and an HMXB. Effectively, we have made the assumption that all Pop III stars end their lives as a BH, without any SN explosion preceding their deaths. If a Pop III star, however, has a mass in the range of $140-260 M_{\odot}$, then it would die as a PISN, exerting a strong mechanical feedback on the surrounding primordial gas, enriching it with the metals produced before and during the explosion. We are able to directly compare our results to those of Greif et al. (2010), where PISN enrichment of the IGM, stellar radiative feedback, chemical mixing, and metal-line cooling were included, because all simulations start 

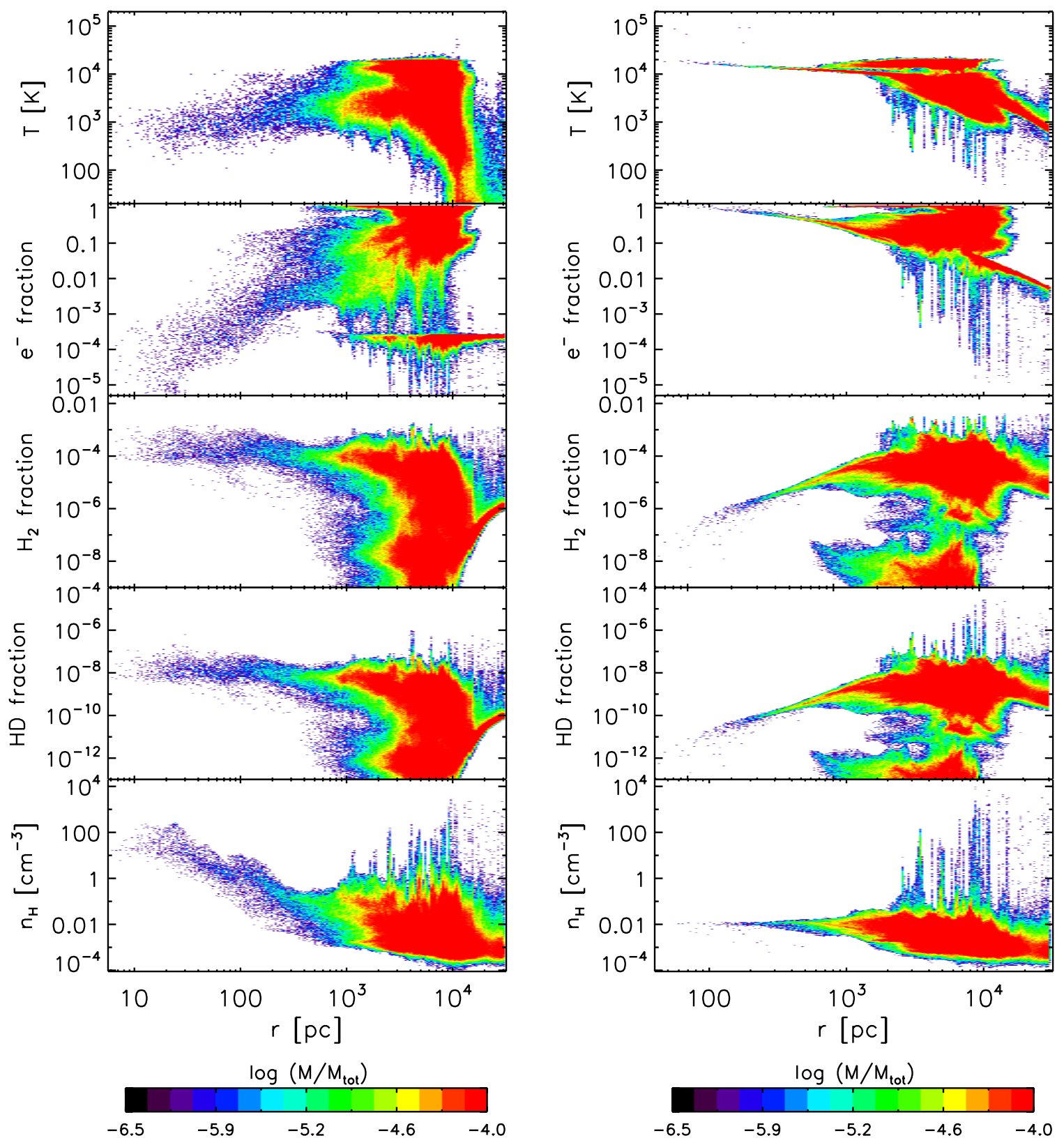

Figure 13. Impact of HMXB feedback. We compare the protogalactic gas properties in simulations BHN (left) and BHB (right) at $z=15$, similar to Figure 11, but now extending to $30 \mathrm{kpc}$ to illustrate the HMXB feedback effect not only on the gas within the host halo, but also on the extended IGM.

(A color version of this figure is available in the online journal.)

from the same initial conditions. The underlying DM structure was thus identical, providing us with an ideal laboratory to study the impact of variations in the baryonic physics on the assembly process of the first galaxies.

We find that the gas properties within the virial radius of the host halo in our BHN simulation at $z \sim 10$ are very similar to those in the Greif et al. (2010) PISN simulation, except that the central gas in the latter simulation is enriched to $Z \sim 10^{-3} Z_{\odot}$. It is, however, still unclear whether such enrichment level is sufficient to enable the transition from Pop III to Pop II star formation, because the critical metallicity threshold for this transition is still a matter of debate. The additional heating from the central BH in our BHS run, on the other hand, significantly enhances molecule formation, possibly by up to two orders of magnitude, and the gas might be able to further fragment. Which feedback is dominant in shaping second-generation star formation in the first galaxies? The answer will strongly depend on the IMF of the first generation of stars and on the detailed physics of the transition from the predicted top-heavy IMF for Pop III to the more normal IMF in already metal-enriched gas.

\section{SUMMARY AND CONCLUSIONS}

We have studied how the assembly of a primordial galaxy is affected by the radiative feedback from an accreting, isolated stellar-mass BH and an HMXB, which are two possible end products of Pop III star formation. To accomplish this, we have carried out three cosmological simulations which selfconsistently account for the radiation from individual Pop III stars and from a central BH X-ray source. In particular, we have focused on the early growth of a Pop III BH remnant at high redshifts and on the role of $\mathrm{BH}$ feedback in shaping subsequent 


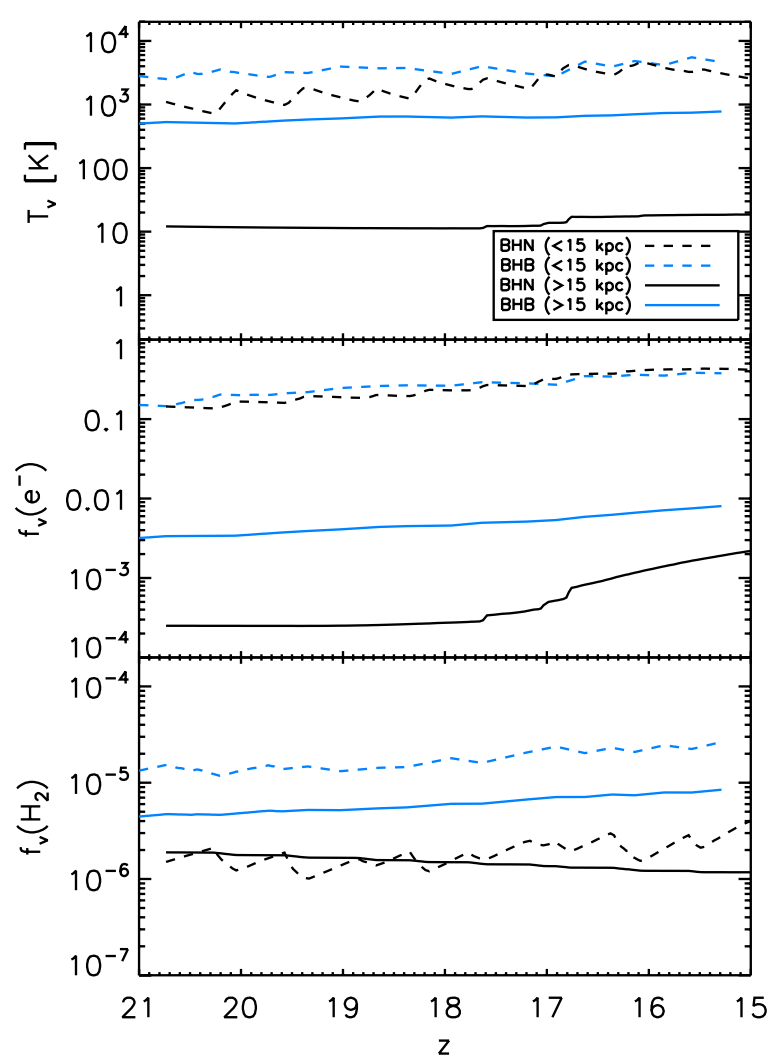

Figure 14. Impact of HMXB feedback on IGM. We show the volume-averaged temperature, electron fraction, and $\mathrm{H}_{2}$ fraction vs. redshift in simulations BHN and $\mathrm{BHB}$. We show these quantities separately for radial distances smaller and larger than $15 \mathrm{kpc}$, beyond which the feedback from Pop III stars is not included. Hence, the feedback effect from the HMXB on the distant IGM becomes clearly apparent.

(A color version of this figure is available in the online journal.)

star formation in the emerging first galaxy at $z \sim 10$. Our main results and implications can be summarized as follows.

1. We have shown that locally, as opposed to any global effect on the large-scale IGM, the feedback from an isolated, accreting $\mathrm{BH}$ is very efficient, leading to a strong suppression of the early growth of the seed BH. Without such feedback, the growth rate quickly reaches near-Eddington values. Our results imply the following important consequence. A widely adopted assumption for the formation of early SMBHs, whose presence is inferred from QSO observations at $z \sim 6$, is that their growth begins with a Pop III seed $\mathrm{BH}$. If this assumption were correct, then quenching of $\mathrm{BH}$ accretion would be required to avoid an overabundance of SMBHs at low redshifts (e.g., Bromm \& Loeb 2003; Trenti \& Stiavelli 2007). Without such quenching, one would also run into inconsistencies with the low number density of quasars, $(6 \pm 2) \times 10^{-10} \mathrm{Mpc}^{-3}$ at $z \sim 6$, assuming $H_{0}=65 \mathrm{~km} \mathrm{~s}^{-1} \mathrm{Mpc}^{-1}, \Omega_{m}=0.35$, and $\Omega_{\Lambda}=0.65$ (Fan et al. 2004). We suggest that the radiative feedback from accreting BHs plays a key role in suppressing early $\mathrm{BH}$ growth, thus constraining models for SMBH formation.

However, in this study we have tracked only an isolated seed $\mathrm{BH}$, born in a halo of $5 \times 10^{5} \mathrm{M}_{\odot}$ that virialized at $z=28$. Alternatively, a $\mathrm{BH}$ seed could form significantly earlier, in a halo corresponding to rare, very high $\sigma$ peaks, thus providing a head start to the buildup process. A different growth-boosting mechanism has been suggested by Volonteri \& Rees (2005), arguing that Pop III remnant BHs, hosted in halos with a virial temperature of $T_{\text {vir }} \gtrsim$ $10^{4} \mathrm{~K}$ at $z \sim 25$, corresponding to peaks of $4 \sigma$ or above, may be able to experience stable supercritical accretion owing to effective atomic hydrogen line cooling (see also Li 2011). It is still challenging to numerically test this scenario because of the prohibitive computational expense to capture such rare massive halos and follow their evolution.

2. Despite the extremely small accretion rates onto the isolated $\mathrm{BH}$, the corresponding radiative feedback on the thermal and chemical evolution of the gas in the immediate surroundings, at scales of $\lesssim 100 \mathrm{pc}$, is significant. The $\mathrm{BH}$ feedback establishes two distinct thermodynamic branches in the central region of the emerging galaxy: a hot phase with $T \sim 10^{4} \mathrm{~K}$ and high ionization fraction, as well as a warm one with $T \sim 10^{3} \mathrm{~K}$ and partially ionized conditions. In the latter phase, molecular hydrogen formation is favored, leading to an elevated fraction of $f_{\mathrm{H}_{2}} \gtrsim 10^{-2}$, two orders of magnitude higher than in the absence of the radiation from a $\mathrm{BH}$. This enhanced $\mathrm{H}_{2}$ fraction could promote second-generation star formation within the emerging first galaxies, possibly resulting in a burst-like mode. However, we do not include the effect of LW molecule-dissociating photons emitted by the soft (MCD) component of the accreting $\mathrm{BH}$. Our $\mathrm{H}_{2}$ and $\mathrm{HD}$ molecule abundances are therefore upper limits. In future work, we will explore the impact of $\mathrm{LW}$ radiation generated by $\mathrm{BH}$ accretion.

What would be the characteristics of the stars formed out of such metal-free gas with a significantly enhanced electron fraction, the so-called Pop III.2 stars (O'Shea et al. 2008)? This issue is far from being settled. Existing studies suggest that the elevated $\mathrm{H}_{2}$ and $\mathrm{HD}$ fractions will lead to rapid cooling, resulting in smaller mass stars, of the order of $\sim 10 M_{\odot}$, compared to the canonical value of $\sim 100 M_{\odot}$ for Pop III.1 stars formed in quasi-neutral minihalos (e.g., O'Shea et al. 2005; Johnson \& Bromm 2006; Yoshida et al. 2007b). However, the suite of simulations carried out by Clark et al. (2011a), who considered turbulent fragmentation in primordial gas, has indicated, somewhat counterintuitively, that the second-generation Pop III. 2 stars were likely more massive than the Pop III.1 stars formed in the pristine minihalos that are unaffected by prior star formation. This can be understood by the inability of the gas in the Pop III. 2 case to reach the CMB temperature floor via HD cooling under realistic halo conditions.

3. The feedback from an efficiently radiating HMXB is very strong locally, and moderately important globally. Locally, the effect on the surrounding primordial gas is to heat it to high temperatures of $\gtrsim 10^{4} \mathrm{~K}$ and to fully ionize it. The corresponding strong photoevaporative outflow suppresses central gas densities, thus preventing any subsequent star formation within the emerging galaxy. Our results imply that once a halo of $\sim 10^{6} M_{\odot}$ harbors an HMXB, the ensuing strong radiative feedback will delay the condensation of gas in the atomic cooling halo, possibly leading to a decrease in the number of first galaxies at a given epoch. While we explore in this paper the impact of a continuously radiating $\mathrm{HMXB}$, the strength of the effect will depend on the realistic abundance and lifetime of such HMXB sources at high redshifts. The highly penetrating X-ray photons emitted by the HMXB are able to effectively heat and partially ionize the distant IGM as well, and as a result catalyze the formation of molecular hydrogen over large 
volumes. We have shown that the global star formation rate in the presence of HMXB feedback is, at least at early times, higher than in the runs without it. We assume that the broad protostellar mass function seen in recent highresolution simulations suggesting that primordial stars have less extreme masses is likely to increase the possibility of HMXBs. However, the mapping between the protostellar fragments, as represented by sink particles, to the final Pop III stars is still incompletely understood. Improved simulations should soon be able to further elucidate this key issue (see Greif et al. 2012).

To sum up, the radiative feedback from accreting $\mathrm{BH}$ sources, either isolated or in a binary system, has a dramatic impact on the surrounding gas within the first galaxy. The alternative, where no BH feedback is present, but where the gas is processed by a preceding PISN explosion, is less affected in the long run. However, it is likely that the enhanced cooling due to metals and dust will only become effective at densities that are higher than can be resolved in these simulations. One possible consequence of metal and dust cooling might be an enhanced star formation efficiency, potentially enabling the formation of a bona fide Pop II, stellar cluster inside the first galaxies (Clark et al. 2008; Tsuribe \& Omukai 2008; Dopcke et al. 2011). To better understand such Pop II star formation, we need to follow the subsequent collapse of the dense, metal-enriched gas with much higher numerical resolution in future simulations.

V.B. and M.M. acknowledge support from NSF grants AST-0708795 and AST-1009928 and NASA ATFP grant NNX09AJ33G. V.B. also acknowledges support from JPL Research Support Agreement 1354840. The simulations were carried out at the Texas Advanced Computing Center (TACC). V.B. thanks the Max-Planck-Institut für Astrophysik for its hospitality during part of the work on this paper. R.S.K. and S.C.O.G. acknowledge support by contract research Internationale Spitzenforschung II of the Baden-Württemberg Stiftung (grant P-LS-SPII/18), from the German Bundesministerium für Bildung und Forschung via the ASTRONET project STAR FORMAT (grant 05A09VHA), from the Deutsche Forschungsgemeinschaft (DFG) under grants KL1358/10 and KL1358/11, via the collaborative research project SFB 881 "The Milky Way Galaxy," and via the priority program SPP 1573 "Physics of the Interstellar Medium" (KL 1358/14).

\section{REFERENCES}

Abel, T., Anninos, P., Zhang, Y., \& Norman, M. L. 1997, New Astron., 2, 181 Abel, T., Bryan, G. L., \& Norman, M. L. 2002, Science, 295, 93

Abel, T., Wise, J. H., \& Bryan, G. L. 2007, ApJ, 659, L87

Alvarez, M. A., Bromm, V., \& Shapiro, P. R. 2006, ApJ, 639, 621

Alvarez, M. A., Wise, J. H., \& Abel, T. 2009, ApJ, 701, L133

Barkana, R., \& Loeb, A. 2001, Phys. Rep., 349, 125

Barkat, Z., Rakavy, G., \& Sack, N. 1967, Phys. Rev. Lett., 18, 379

Bate, M. R., Bonnell, I. A., \& Price, N. M. 1995, MNRAS, 277, 362

Bate, M. R., \& Burkert, A. 1997, MNRAS, 288, 1060

Beers, T. C., \& Christlieb, N. 2005, ARA\&A, 43, 531

Blandford, R. D., \& Begelman, M. C. 1999, MNRAS, 303, L1

Blandford, R. D., \& Begelman, M. C. 2004, MNRAS, 349, 68

Bondi, H., \& Hoyle, F. 1944, MNRAS, 104, 273

Bromm, V., Coppi, P. S., \& Larson, R. B. 1999, ApJ, 527, L5

Bromm, V., Coppi, P. S., \& Larson, R. B. 2002, ApJ, 564, 23

Bromm, V., Ferrara, A., Coppi, P. S., \& Larson, R. B. 2001a, MNRAS, 328, 969

Bromm, V., Kudritzki, R. P., \& Loeb, A. 2001b, ApJ, 552, 464

Bromm, V., \& Larson, R. B. 2004, ARA\&A, 42, 79

Bromm, V., \& Loeb, A. 2003, ApJ, 596, 34

Bromm, V., \& Yoshida, N. 2011, ARA\&A, 49, 373
Bromm, V., Yoshida, N., Hernquist, L., \& McKee, C. F. 2009, Nature, 459, 49 Ciardi, B., \& Ferrara, A. 2005, Space Sci. Rev., 116, 625

Clark, P. C., Glover, S. C. O., \& Klessen, R. S. 2008, ApJ, 672, 757

Clark, P. C., Glover, S. C. O., Klessen, R. S., \& Bromm, V. 2011a, ApJ, 727, 110

Clark, P. C., Glover, S. C. O., Smith, R. J., et al. 2011b, Science, 331, 1040

Dopcke, G., Glover, S. C. O., Clark, P. C., \& Klessen, R. S. 2011, ApJ, 729, L3 Draine, B. T., \& Bertoldi, F. 1996, ApJ, 468, 269

Dray, L. M. 2006, MNRAS, 370, 2079

Fan, X., Hennawi, J. F., Richards, G. T., et al. 2004, AJ, 128, 515

Fan, X., Strauss, M. A., Richards, G. T., et al. 2006, AJ, 131, 1203

Furlanetto, S. R., Oh, S. P., \& Briggs, F. H. 2006, Phys. Rep., 433, 181

Furlanetto, S. R., \& Stoever, S. J. 2010, MNRAS, 404, 1869

Glover, S. C. O., \& Brand, P. W. J. L. 2003, MNRAS, 340, 210

Greif, T. H., Bromm, V., Clark, P. C., et al. 2012, arXiv:1202.5552

Greif, T. H., Glover, S. C. O., Bromm, V., \& Klessen, R. S. 2010, ApJ, 716, 510

Greif, T. H., Johnson, J. L., Klessen, R. S., \& Bromm, V. 2008, MNRAS, 387, 1021

Greif, T. H., Johnson, J. L., Klessen, R. S., \& Bromm, V. 2009, MNRAS, 399, 639

Greif, T. H., Springel, V., White, S. D. M., et al. 2011, ApJ, 737, 75

Grimm, H.-J., Gilfanov, M., \& Sunyaev, R. 2003, MNRAS, 339, 793

Haiman, Z. 2011, Nature, 472, 47

Haiman, Z., \& Loeb, A. 2001, ApJ, 552, 459

Haiman, Z., Thoul, A. A., \& Loeb, A. 1996, ApJ, 464, 523

Heger, A., Fryer, C. L., Woosley, S. E., Langer, N., \& Hartmann, D. H. 2003, ApJ, 591,288

Heger, A., \& Woosley, S. E. 2002, ApJ, 567, 532

Hernquist, L., \& Springel, V. 2003, MNRAS, 341, 1253

Hosokawa, T., Omukai, K., Yoshida, N., \& Yorke, H. W. 2011, Science, 334, 1250

Jappsen, A.-K., Glover, S. C. O., Klessen, R. S., \& Mac Low, M.-M. 2007, ApJ, 660,1332

Jappsen, A.-K., Klessen, R. S., Glover, S. C. O., \& Mac Low, M.-M. 2009a, ApJ, 696, 1065

Jappsen, A.-K., Klessen, R. S., Larson, R. B., Li, Y., \& Mac Low, M.-M. 2005, A\&A, 435, 611

Jappsen, A.-K., Mac Low, M.-M., Glover, S. C. O., Klessen, R. S., \& Kitsionas, S. 2009b, ApJ, 694, 1161

Joggerst, C. C., Almgren, A., Bell, J., et al. 2010, ApJ, 709, 11

Johnson, J. L., \& Bromm, V. 2006, MNRAS, 366, 247

Johnson, J. L., \& Bromm, V. 2007, MNRAS, 374, 1557

Johnson, J. L., Greif, T. H., \& Bromm, V. 2007, ApJ, 665, 85

Johnson, J. L., Khochfar, S., Greif, T. H., \& Durier, F. 2011, MNRAS, 410, 919

Karlsson, T., Johnson, J. L., \& Bromm, V. 2008, ApJ, 679, 6

Kitayama, T., Yoshida, N., Susa, H., \& Umemura, M. 2004, ApJ, 613, 631

Komatsu, E., Dunkley, J., Nolta, M. R., et al. 2009, ApJS, 180, 330

Komatsu, E., Smith, K. M., Dunkley, J., et al. 2011, ApJS, 192, 18

Koushiappas, S. M., Bullock, J. S., \& Dekel, A. 2004, MNRAS, 354, 292

Krumholz, M. R., McKee, C. F., \& Klein, R. I. 2005, ApJ, 618, 757

Kuhlen, M., \& Madau, P. 2005, MNRAS, 363, 1069

Latif, M. A., Zaroubi, S., \& Spaans, M. 2011, MNRAS, 411, 1659

Li, Y. 2011, arXiv:1109.3442

Li, Y., Hernquist, L., Robertson, B., et al. 2007, ApJ, 665, 187

Linden, T., Kalogera, V., Sepinsky, J. F., et al. 2010, ApJ, 725, 1984

Machacek, M. E., Bryan, G. L., \& Abel, T. 2003, MNRAS, 338, 273

Madau, P., \& Rees, M. J. 2001, ApJ, 551, L27

Madau, P., Rees, M. J., Volonteri, M., Haardt, F., \& Oh, S. P. 2004, ApJ, 604, 484

Majid, W. A., Lamb, R. C., \& Macomb, D. J. 2004, ApJ, 609, 133

Mapelli, M., Colpi, M., \& Zampieri, L. 2009, MNRAS, 395, L71

Miller, J. M., Fabbiano, G., Miller, M. C., \& Fabian, A. C. 2003, ApJ, 585, L37

Milosavljević, M., Bromm, V., Couch, S. M., \& Oh, S. P. 2009a, ApJ, 698, 766

Milosavljević, M., Couch, S. M., \& Bromm, V. 2009b, ApJ, 696, L146

Mirabel, I. F., Dijkstra, M., Laurent, P., Loeb, A., \& Pritchard, J. R. 2011, A\&A, 528, A149

Mitsuda, K., Inoue, H., Koyama, K., et al. 1984, PASJ, 36, 741

Nakamura, F., \& Umemura, M. 2001, ApJ, 548, 19

Narayan, R., \& Yi, I. 1994, ApJ, 428, L13

Narayan, R., \& Yi, I. 1995, ApJ, 452, 710

Oh, S. P. 2001, ApJ, 553, 499

Oh, S. P., \& Haiman, Z. 2002, ApJ, 569, 558

Omukai, K. 2000, ApJ, 534, 809

Omukai, K., \& Palla, F. 2003, ApJ, 589, 677

O'Shea, B. W., Abel, T., Whalen, D., \& Norman, M. L. 2005, ApJ, 628, L5

O'Shea, B. W., McKee, C. F., Heger, A., \& Abel, T. 2008, in AIP Conf. Proc. 990, First Stars III, ed. B. W. O'Shea \& A. Heger (Melville, NY: AIP), D13 
O’Shea, B. W., \& Norman, M. L. 2007, ApJ, 654, 66

Osterbrock, D. E., \& Ferland, G. J. (ed.) 2006, Astrophysics of Gaseous Nebulae and Active Galactic Nuclei (Sausalito, CA: Univ. Science Books)

Park, K., \& Ricotti, M. 2011, ApJ, 739, 2

Park, K., \& Ricotti, M. 2012, ApJ, 747, 9

Pelupessy, F. I., Di Matteo, T., \& Ciardi, B. 2007, ApJ, 665, 107

Prieto, J., Padoan, P., Jimenez, R., \& Infante, L. 2011, ApJ, 731, L38

Regan, J. A., \& Haehnelt, M. G. 2009, MNRAS, 393, 858

Ricotti, M., Gnedin, N. Y., \& Shull, J. M. 2001, ApJ, 560, 580

Ricotti, M., Gnedin, N. Y., \& Shull, J. M. 2002, ApJ, 575, 49

Ricotti, M., \& Ostriker, J. P. 2004, MNRAS, 352, 547

Ripamonti, E., Mapelli, M., \& Zaroubi, S. 2008, MNRAS, 387, 158

Schaerer, D. 2002, A\&A, 382, 28

Schleicher, D. R. G., Spaans, M., \& Glover, S. C. O. 2010, ApJ, 712, L69

Schneider, R., Ferrara, A., Natarajan, P., \& Omukai, K. 2002, ApJ, 571, 30

Shang, C., Bryan, G. L., \& Haiman, Z. 2010, MNRAS, 402, 1249

Shapiro, P. R., \& Kang, H. 1987, ApJ, 318, 32

Shu, F. H., Lizano, S., Galli, D., Cantó, J., \& Laughlin, G. 2002, ApJ, 580, 969

Shull, J. M., \& van Steenberg, M. E. 1985, ApJ, 298, 268

Smith, R. J., Glover, S. C. O., Clark, P. C., Greif, T., \& Klessen, R. S. 2011, MNRAS, 414, 3633

Soria, R. 2007, Ap\&SS, 311, 213
Springel, V. 2005, MNRAS, 364, 1105

Stacy, A., Greif, T. H., \& Bromm, V. 2010, MNRAS, 403, 45

Stacy, A., Greif, T. H., \& Bromm, V. 2012, MNRAS, 422, 290

Tanaka, T., \& Haiman, Z. 2009, ApJ, 696, 1798

Tegmark, M., Silk, J., Rees, M. J., et al. 1997, ApJ, 474, 1

Thomas, R. M., \& Zaroubi, S. 2008, MNRAS, 384, 1080

Trenti, M., \& Stiavelli, M. 2007, ApJ, 667, 38

Tsuribe, T., \& Omukai, K. 2008, ApJ, 676, L45

Turk, M. J., Abel, T., \& O'Shea, B. 2009, Science, 325, 601

Valdés, M., \& Ferrara, A. 2008, MNRAS, 387, L8

Volonteri, M., \& Rees, M. J. 2005, ApJ, 633, 624

Volonteri, M., \& Rees, M. J. 2006, ApJ, 650, 669

Whalen, D., Abel, T., \& Norman, M. L. 2004, ApJ, 610, 14

Wheeler, J. C., \& Johnson, V. 2011, ApJ, 738, 163

Wise, J. H., \& Abel, T. 2007, ApJ, 665, 899

Wise, J. H., \& Abel, T. 2008, ApJ, 685, 40

Wise, J. H., Turk, M. J., Norman, M. L., \& Abel, T. 2012, ApJ, 745, 50

Wolcott-Green, J., \& Haiman, Z. 2011, MNRAS, 412, 2603

Yoshida, N., Abel, T., Hernquist, L., \& Sugiyama, N. 2003, ApJ, 592, 645

Yoshida, N., Oh, S. P., Kitayama, T., \& Hernquist, L. 2007a, ApJ, 663, 687

Yoshida, N., Omukai, K., \& Hernquist, L. 2007b, ApJ, 667, L117

Yoshida, N., Omukai, K., Hernquist, L., \& Abel, T. 2006, ApJ, 652, 6

Zaroubi, S., Thomas, R. M., Sugiyama, N., \& Silk, J. 2007, MNRAS, 375, 1269 